\title{
Nonlooseness of nonloose knots
}

\author{
KENNETH L BAKER \\ SINEM ONARAN
}

\begin{abstract}
A Legendrian or transverse knot in an overtwisted contact 3-manifold is nonloose if its complement is tight and loose if its complement is overtwisted. We define three measures of the extent of nonlooseness of a nonloose knot and show they are distinct.
\end{abstract}

53D10, 57M27

\section{Introduction}

A contact 3-manifold $(M, \xi)$ is overtwisted if it contains an overtwisted disk and tight otherwise. A Legendrian or transverse knot $K$ in an overtwisted contact 3-manifold is called nonloose (or exceptional as in Eliashberg and Fraser [11]) if the restriction of the contact manifold to its complement is tight. If instead the complement is overtwisted, then $K$ is called loose. That is, $K$ is nonloose if it intersects every overtwisted disk, while $K$ is loose if it is disjoint from some overtwisted disk. In this article we develop and examine notions of the extent of nonlooseness of Legendrian and transverse knots in overtwisted contact structures. Throughout, our ambient 3-manifolds will be closed, compact, connected and oriented and our contact structures will be cooriented.

For an unoriented Legendrian knot $L$ in a closed overtwisted contact 3-manifold $(M, \xi)$ we define three invariants, two geometric and one algebraic:

- The depth of $L, d(L)$, is the minimum of $|L \cap D|$ over all overtwisted disks $D$ in $M$.

- The tension of $L, t(L)$, is the minimum number of stabilizations required to loosen $L$.

- The order of $L, \bar{o}(L)$, is the sum of the orders of the $U$-torsion of the LOSS invariant $\mathfrak{L}$ (defined by Lisca, Ozsváth, Stipsicz and Szabó in [27]) of the two orientations on $L$. Presently this is only defined when $L$ is nullhomologous.

If $L$ is loose, then all three of these are 0 . If $L$ is nonloose then both its depth and the tension are nonzero by definition, though its order may be 0 . 
Theorem 1.0.1 If $L$ is a Legendrian knot in an overtwisted contact 3-manifold, then

$$
\bar{o}(L) \leq t(L) \leq d(L),
$$

where we only consider $\bar{o}(L)$ if $L$ is nullhomologous.

Proof The second inequality is Lemma 4.1.6. When $L$ is also nullhomologous, the first inequality is Lemma 4.3.4.

Indeed, these three invariants are all distinct.

Theorem 1.0.2 There exist nonloose Legendrian knots $L$ with

$$
1=t(L)<d(L) .
$$

Sketch of proof The following two theorems give a surgery characterization of nonloose Legendrian knots with depth 1 and a surgery construction of nonloose Legendrian knots with tension 1. In particular, if $(+1)$-surgery on a nondestabilizable Legendrian knot $L$ in $\left(S^{3}, \xi_{\text {std }}\right)$ satisfying $\operatorname{tb}(L) \leq-2, \operatorname{rot}(L)<0$, $-\chi(L)<-(\operatorname{rot}(L)+\operatorname{tb}(L)+2)$ is overtwisted, then the surgery dual is a nonloose Legendrian knot $L^{*}$ with $1=t\left(L^{*}\right)<d\left(L^{*}\right)$. Etnyre and Honda [15] show there is a Legendrian torus knot satisfying these classical constraints that, according to Lisca and Stipsicz [29], has an overtwisted (+1)-surgery. A more detailed proof is given after the proof of Theorem 4.1.8.

Theorem 4.1.7 Suppose $(+1)$-surgery on a Legendrian knot $L$ with tight complement yields an overtwisted manifold with surgery dual knot $L^{*}$. Then $d\left(L^{*}\right)=1$ if and only if $L$ is a stabilization.

Theorem 4.1.8 Suppose $(+1)$-surgery on a Legendrian knot $L$ in $\left(S^{3}, \xi_{\text {std }}\right)$ yields an overtwisted manifold with surgery dual knot $L^{*}$. If $\operatorname{tb}(L)<-1, \operatorname{rot}(L)<0$ and $\operatorname{tb}(L)+\operatorname{rot}(L)+2<\chi(L)$, then $t\left(L^{*}\right)=1$.

Theorem 1.0.3 A nonloose Legendrian unknot $L$ satisfies

$$
\bar{o}(L)=0 \quad \text { while } \quad t(L)=d(L)=1 .
$$

Proof Lemma 4.1.9 shows any nonloose Legendrian unknot has depth 1 and hence, by Theorem 1.0.1 (or just Lemma 4.1.6), also tension 1. Corollary 4.3.8 shows they all have order 0 . 
The proof above relies upon Theorem 3.3.1, the characterization of nonloose unknots in $S^{3}$ of Eliashberg and Fraser [11], and Corollary 3.3.3, its implication for other manifolds. The key step to Lemma 4.1.9 is the application of Theorem 4.1.7 to the surgery diagrams given by Plamenevskaya [34] of these nonloose unknots in $S^{3}$. Corollary 4.3.8 is a consequence of the following proposition which exploits the behavior of the LOSS invariant under stabilizations. Here we state it for just $\bar{o}$, but the actual proposition addresses $\mathfrak{L}$ and related invariants as well.

Proposition 4.3.7 If a nullhomologous knot type $\mathcal{K}$ has a lower bound on the ThurstonBennequin invariants of its nonloose Legendrian representatives in a given overtwisted contact structure, then $\bar{o}(L)=0$ for each Legendrian representative $L$.

We also consider refinements of the above invariants for oriented Legendrian knots and their analogues for transverse knots. The binding of an open book is naturally a transverse link in the contact structure supported by the open book. If the open book is a negative Hopf stabilization of another open book, then the binding has depth 1 .

Theorem 5.2.3 Assume an open book with connected binding is a negative Hopf stabilization. Then the binding $T$, as the nonloose transverse knot in the overtwisted contact structure the open book supports, has $d(T)=t(T)=1$.

Dymara [9] and Eliashberg and Fraser [11] established fundamentals about nonloose knots with a focus on nonloose unknots. Dymara attributes Świątowski with a Bennequin-type inequality for nonloose Legendrian knots which we recall in Theorem 3.2.1. In his study of the coarse classification of nonloose Legendrian and transverse knots, Etnyre [14] gives the associated Bennequin inequality for nonloose transverse knots, Theorem 3.2.2. These two theorems are both for nullhomologous knots; in the vein of Baker and Etnyre [1], we extend them to rationally nullhomologous knots.

Theorem 5.2.3 For a nonloose rationally nullhomologous Legendrian knot $L$ of homological order $r$ with rational Seifert surface $\Sigma$,

$$
-\left|\operatorname{tb}_{\mathbb{Q}}(L)\right|+\left|\operatorname{rot}_{\mathbb{Q}}(L)\right| \leq-\frac{1}{r} \chi(\Sigma) .
$$

Theorem 3.2.4 For a rationally nullhomologous nonloose transverse knot $T$ of homological order $r$ with rational Seifert surface $\Sigma$,

$$
\mathrm{sl}_{\mathbb{Q}}(T) \leq-\frac{1}{r} \chi(\Sigma)
$$




\subsection{Outline}

We recall the basic concepts and tools for dealing with contact structures and the Legendrian and transverse knots in them in Section 2 while Section 3 covers the basics of overtwisted manifolds and nonloose knots. Section 4 develops our invariants and main results for nonloose Legendrian knots; Section 5 addresses nonloose transverse knots. We conclude with a handful of problems and questions in Section 6.

Acknowledgements We would like to thank Keiko Kawamuro and David Shea VelaVick for helpful conversations and Steven Sivek for his input. Kenneth Baker was partially supported by grant number 209184 from the Simons Foundation. Sinem Onaran is supported by the Scientific and Technological Research Council of Turkey (grant TUBITAK 3501-\#112T994) and she thanks the Max Planck Institute for Mathematics, Bonn for their hospitality.

\section{Preliminaries}

\subsection{Contact structures}

A contact structure $\xi$ on a 3 -manifold $M$ is a nowhere integrable 2-plane field, and the pair $(M, \xi)$ forms a contact 3-manifold. Locally, $\xi$ is orientation-preserving diffeomorphic to ker $\alpha$ for some 1 -form $\alpha$ satisfying $\alpha \wedge d \alpha \neq 0$. We restrict attention to positive contact structures; those for which $\alpha \wedge d \alpha>0$. By Darboux's theorem (see Geiges [19]) every point in a (positive) contact manifold $(M, \xi)$ has a neighborhood admitting an orientation-preserving diffeomorphism to an open subset of $\left(\mathbb{R}^{3}, \xi_{\text {std }}\right)$; the standard contact structure on $\mathbb{R}^{3}$, where $\xi_{\text {std }}=\operatorname{ker}(d z-y d x)$.

\subsection{Legendrian and transverse knots}

A particular smooth embedding of an oriented knot $K$ in a contact 3-manifold $(M, \xi)$ is Legendrian if its tangent vectors lie in the contact planes: $T_{p} K \subset \xi_{p}$ for every $p \in K$. On the other hand, the knot $K$ is transverse if its tangent vectors are not in the contact planes: $T_{p} K \oplus \xi_{p} \cong T_{p} M$ for every $p \in K$. The coorientation of $\xi$ naturally orients $K$, and hence we always regard transverse knots as oriented knots.

An isotopy through Legendrian embeddings is a Legendrian isotopy, and an isotopy through transverse embeddings is a transverse isotopy. A Legendrian knot is a Legendrian isotopy equivalence class, and a transverse knot is a transverse isotopy equivalence class. A good reference for the fundamentals of Legendrian knots and transverse knots is Etnyre [13]. 
2.2.1 Classical invariants The most basic invariant of a Legendrian or transverse knot is its topological knot type.

The contact structure endows a Legendrian knot $L$ with a natural framing $\lambda_{\xi}$ called its contact framing. Given a surface $\Sigma$ embedded in $(M, \xi)$ that contains $L$ (or even just properly embedded in $M-\mathcal{N}(L)$ and radially extended to $L$ in $\mathcal{N}(L)$ ), the twist number of $\Sigma$ along $L$ relative to $\xi$ measures how $\Sigma$ twists along $L$ relative to $\xi$. That is, $\operatorname{tw}_{\xi}(L, \Sigma)$ is the slope $(p / q)$ of the curve $\sigma=\Sigma \cap \partial \mathcal{N}(L)$, where for some orientation we have $[\sigma]=p[\mu]+q\left[\lambda_{\xi}\right]$. Here we view the meridian $\mu$ of $L$ and the framing $\lambda_{\xi}$ in the boundary of a regular neighborhood of $L, \partial \mathcal{N}(L)$ and orient them so that if $L$ is oriented to be parallel to $\lambda_{\xi}$ then $L$ links $\mu$ once positively.

Caution When $\Sigma$ is an orientable surface containing $L$, it is common to measure the twisting of $\xi$ along $L$ relative to $\Sigma$ instead (as in the definition of the ThurstonBennequin invariant below). This means our twist number has its sign opposite from what may be more traditional.

The Thurston-Bennequin invariant $\operatorname{tb}(L)$ of a nullhomologous Legendrian knot $L$ is the discrepancy between this contact framing and the framing induced by its Seifert surfaces; if $\Sigma$ is a Seifert surface for $L$ then $\operatorname{tb}(L)=-\operatorname{tw}_{\xi}(L, \Sigma)$. The rotation number $\operatorname{rot}(L)$ of an oriented nullhomologous Legendrian knot is the winding number of $T L$ after trivializing the contact structure along a Seifert surface. The self-linking number $\operatorname{sl}(T)$ of a nullhomologous transverse knot $T$ is the linking number of $T$ with a push-off of $T$ in the direction of a nowhere-zero section over $T$ after trivializing the contact structure along a Seifert surface. These are the "classical" invariants of Legendrian and transverse knots (for further details, see [13] for example). These invariants have been generalized to rational versions for rationally nullhomologous knots (see the first-named author and Etnyre [1] and Öztürk [33]) and to relative versions for any knot in relation to a chosen homologous knot (see Chernov [4]). We will use the rational versions $\operatorname{tb}_{\mathbb{Q}}$ and $\operatorname{rot}_{\mathbb{Q}}$ in this article.

2.2.2 Stabilizations Let $L$ be an oriented Legendrian knot in a contact 3-manifold. By Darboux's theorem (see [19]) for each point on $L$ there is a neighborhood $N$ with contactomorphism to $\left(\mathbb{R}^{3}, \xi_{\text {std }}\right)$ sending $L \cap N$ to the $x$-axis. In this manner we locally represent $L$ with the front diagram (the projection to the $x z$-plane) as in the left-hand side of Figure 1. The modification of $L$ to another Legendrian knot $L_{+}$as shown in the top right-hand side of Figure 1 is called a positive stabilization of $L$. Similarly, $L_{-}$shown in the lower right-hand side of Figure 1 is a negative stabilization of $L$. We will also write $L_{+a,-b}$ to indicate the Legendrian knot $L$ with $a$ positive stabilizations and $b$ negative stabilizations for $a, b \geq 0$. Note it does not matter the order in which these stabilizations are done and it does not matter where along $L$ these 
stabilizations are done; the results are Legendrian isotopic. However, as demonstrated by the contactomorphism of $\xi_{\text {std }}$ resulting from the $\pi$ rotation about the $z$-axis reversing the image of $L$, whether a stabilization is positive or negative depends on the orientation of $L:(-L)_{ \pm}=-\left(L_{\mp}\right)$.

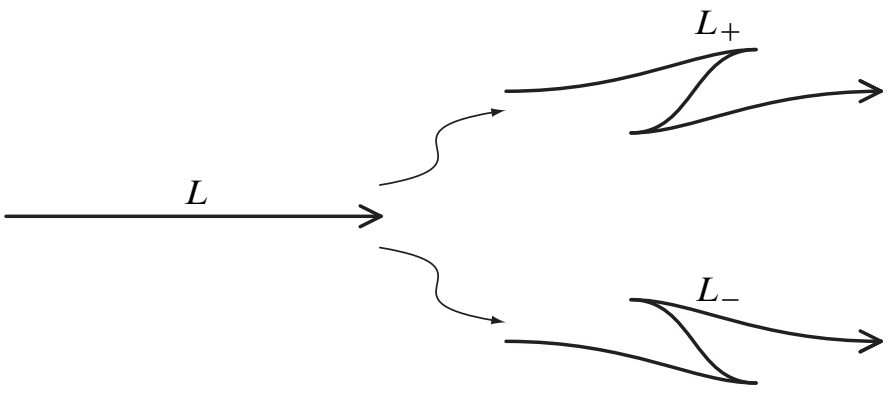

Figure 1

The effect of stabilizations on the classical invariants for Legendrian knots are

$$
\operatorname{tb}\left(L_{ \pm}\right)=\operatorname{tb}(L)-1 \text { and } \operatorname{rot}\left(L_{ \pm}\right)=\operatorname{rot}(L) \pm 1 .
$$

Note that $t b$ is actually an invariant of unoriented Legendrian knots while rot requires an orientation. Indeed, if $-L$ denotes $L$ with the opposite orientation, then $\operatorname{tb}(-L)=$ $\operatorname{tb}(L)$ and $\operatorname{rot}(-L)=-\operatorname{rot}(L)$. (We also note that $\operatorname{tw}_{\xi}\left(L_{ \pm}, \Sigma\right)=\operatorname{tw}_{\xi}(L, \Sigma)+1$.)

2.2.3 Transverse push-offs and Legendrian approximations An oriented Legendrian knot $L$ has a positive and negative transverse push-off, $T_{+}(L)$ and $T_{-}(L)$, defined as follows. Choose a vector field $X$ on $\left.\xi\right|_{L}$ such that at each point $p \in L$ we have the positive oriented basis $\left(X(p), T_{p}(L)\right)$ for $\xi_{p}$. Then a small push-off of $L$ in the direction of $X$ gives the transverse knot $T_{+}(L)$ which is oriented parallel to $L$ by the coorientation of $\xi$. A small push-off of $L$ in the direction of $-X$ gives the transverse knot $T_{-}(L)$ which is oriented parallel to $-L$. We say the positive transverse push-off $T_{+}(L)$ is the transverse push-off. Negative stabilizations of Legendrian knots do not change the transverse push-off, so any invariant of Legendrian knots that is not altered by negative stabilizations gives an invariant of transverse knots.

Note that, by definition, reversing the orientation of a transverse knot does not produce another transverse knot since its orientation no longer agrees with the coorientation of the contact structure.

The contact manifold $\left(\mathbb{R}^{3}, \xi_{\text {cyl }}\right)$ with $\xi_{\text {cyl }}=\operatorname{ker}\left(d z+r^{2} d \theta\right)$ is the cylindrical model of the standard contact structure on $\mathbb{R}^{3}$. There is a contactomorphism $\left(\mathbb{R}^{3}, \xi_{\text {cyl }}\right) \rightarrow$ $\left(\mathbb{R}^{3}, \xi_{\text {std }}\right)$. A regular $\epsilon$-neighborhood of the $z$-axis in this cylindrical model, modulo 
$z \mapsto z+1$, gives a standard solid torus neighborhood for a transverse knot $T$. A Legendrian curve $L$ on a concentric torus that is topologically isotopic to the core $T$ of this solid torus is a Legendrian approximation of $T$. While there are many different Legendrian approximations to $T$, any two have common negative stabilizations. Furthermore, the (positive) transverse push-off of a Legendrian approximation of $T$ is again $T$. See Epstein, Fuchs and Meyer [12] for further details.

\subsection{Convex surfaces}

An embedded surface $\Sigma$ in a contact manifold $(M, \xi)$ is convex if there is an embedded product neighborhood $\Sigma \times(-1,1)$ with $\Sigma=\Sigma \times\{0\}$ such that $\xi$ is preserved by flow in the product direction within this product. This notion is due to Giroux [21]. He also shows that any surface $\Sigma$ embedded in a contact manifold so that each component $L$ of $\partial \Sigma$ is Legendrian with $\operatorname{tw}_{\xi}(L, \Sigma) \geq 0$ admits a $C^{0}$-isotopy fixing its boundary to a convex representative. Such an isotopy may be found in any neighborhood of the surface.

Given an oriented convex surface $\Sigma$ with (possibly empty) Legendrian boundary, let $X$ be a vector field given by a vertical flow in the product direction of a product neighborhood of $\Sigma$ (for example $X=\partial_{t}$ where $t$ is the interval parameter). For each point $x \in \Sigma$ the vector $X(x) \in T_{x} M$ projects to a positive, zero or negative multiple of the coorientation of $\xi_{x}$. The dividing set $\Gamma$ of $\Sigma$ is the properly embedded 1 -manifold consisting of points for which this is the zero multiple, that is $\Gamma=\left\{x \in \Sigma: X(x) \in \xi_{x}\right\}$. Then $\Sigma_{+}$is the component of $\Sigma-\Gamma$ where the multiple is positive and $\Sigma_{-}$is the component where the multiple is negative. If $\Gamma^{\prime}$ is smoothly isotopic to $\Gamma$ in $\Sigma$, there is a smooth isotopy of $\Sigma$, fixing its boundary, through convex surfaces realizing $\Gamma^{\prime}$ as a dividing set.

If $\Sigma$ is a convex surface with dividing set $\Gamma$ and $L$ is a Legendrian knot in $\Sigma$ (such as component of $\partial \Sigma$ ) then $L$ is necessarily transverse to $\Gamma$ and $\operatorname{tw}_{\xi}(L, \Sigma)=\frac{1}{2}|L \cap \Gamma|$.

\subsection{Contact surgery}

A Legendrian knot $L$ has a standard tight neighborhood $\mathcal{N}(L)$ with convex boundary having two dividing curves. Contact surgery of slope $(p / q)$ is a Dehn surgery on $L$ producing a new contact manifold by replacing $\mathcal{N}(L)$ with another tight contact solid torus having the same boundary but with a meridian of slope $(p / q)$ with respect to the contact framing. (If $L$ is nullhomologous, a $(p / q)$-contact surgery is topologically a Dehn surgery of slope $p / q+\operatorname{tb}(L)$.) While generically there are multiple contact solid tori with the required boundary data, this Dehn surgery is unique when $p= \pm 1$. 
See [19] for a more detailed discussion. In this article we primarily concern ourselves with $( \pm 1)$-surgeries.

After $(1 / n)$-surgery on $L$, the core curve of the attached solid torus is again a Legendrian knot $L^{*}$ called the surgery dual. For such surgeries, both $L$ and $L^{*}$ are isotopic through their solid tori to a curve $L^{\prime}$ in $\partial \mathcal{N}(L)$. We say $L^{\prime}$ is a Legendrian push-off of $L$ and the annulus they cobound is a push-off annulus. In the surgered manifold, $L^{\prime}$ may also be viewed as a Legendrian push-off of $L^{*}$. Through this push-off, an orientation on $L$ confers a natural orientation upon $L^{*}$.

\section{Basics on knots in overtwisted contact structures}

\subsection{Overtwisted disks, loose and nonloose knots}

A smoothly embedded disk $D$ with Legendrian boundary such that $\operatorname{tb}(\partial D)=0$ is an overtwisted disk. We orient $D$ so that it induces the orientation on $\partial D$ with $\operatorname{rot}(\partial D)>0$. A contact manifold is overtwisted if it contains an overtwisted disk, and it is tight otherwise.

For a convex overtwisted disk, since $\operatorname{tb}(\partial D)=0$ it follows that the dividing set $\Gamma$ is disjoint from $\partial D$ and hence is a collection of simple closed curves in the interior of $D$.

A standard overtwisted disk is one with a neighborhood contactomorphic to that of $D_{\text {от }}=\{(r, \theta, 0): r \leq \pi\}$ in the contact manifold $\left(\mathbb{R}^{3}, \xi_{\text {от }}\right)$ where $\xi_{\text {от }}=\operatorname{ker}(\cos r d z+$ $r \sin r d \theta)$. This disk is convex and has one dividing curve: use the vector field $X=\partial_{z}$ so that $\Gamma=\{(\pi / 2, \theta, 0)\}$. Observe that by orienting $D_{\text {От }}$ so that with the boundary orientation $\operatorname{rot}\left(\partial D_{\mathrm{OT}}\right)>0$, the origin is a positive elliptic singularity of the characteristic foliation.

Proposition 3.1.1 (Proof of [19, Proposition 4.6.28]) Any overtwisted disk $D$ admits a slight isotopy fixing its boundary to an overtwisted disk $D^{\prime}$ that either is a standard overtwisted disk or properly contains a standard overtwisted disk.

This gives us an immediate corollary.

Corollary 3.1.2 Let $K$ be a Legendrian or transverse knot in an overtwisted contact $3-$ manifold. Then $K$ intersects every overtwisted disk if and only if $K$ intersects every standard overtwisted disk. 
As mentioned in the introduction, a Legendrian or transverse knot $K$ in an overtwisted contact 3-manifold $(M, \xi)$ is nonloose if $K$ intersects every overtwisted disk (so that its complement is tight) and is loose if it is disjoint from some overtwisted disk (so that its complement is overtwisted). Corollary 3.1.2 lets us rephrase this: $K$ is nonloose if it intersects every standard overtwisted disk and loose if it is disjoint from some standard overtwisted disk. Note that while the complement of any knot in a tight manifold is tight, the term nonloose is used to imply that the ambient contact manifold is overtwisted. Furthermore, observe that if some contact surgery on a Legendrian knot is tight then the complement of the knot is tight; this can be a convenient way to detect nonlooseness.

\subsection{Classical invariants and nonloose knots}

If $L$ were a nullhomologous Legendrian knot in a tight contact manifold, then the Bennequin inequality (see Bennequin [3] and Eliashberg [10]) would hold true: $\operatorname{tb}(L)+$ $|\operatorname{rot}(L)| \leq-\chi(L)$. In an overtwisted contact manifold, one may find Legendrian knots that violate this inequality. Loose Legendrian knots of arbitrarily large tb may be constructed by exploiting the overtwisted disks in their complements. On the other hand, nonloose Legendrian knots could potentially violate the Bennequin inequality if $t b$ were positive and sufficiently large.

Theorem 3.2.1 (Świątowski [9; 14]) For a nonloose nullhomologous Legendrian knot $L$ with Seifert surface $\Sigma$,

$$
-|\operatorname{tb}(L)|+|\operatorname{rot}(L)| \leq-\chi(\Sigma) .
$$

Theorem 3.2.2 [14] For a nonloose nullhomologous transverse knot $T$ with Seifert surface $\Sigma$,

$$
\operatorname{sl}(T) \leq-\chi(\Sigma)
$$

This follows from the relation

$$
\operatorname{sl}\left(T_{ \pm}(L)\right)=\operatorname{tb}(L) \mp \operatorname{rot}(L),
$$

where $T_{ \pm}(L)$ denotes the positive and negative transverse push-offs of $L$.

We give an extension of these bounds to rationally nullhomologous nonloose knots modeled on the proof of these given in [14]. See [1] for details on the rational classical invariants and Geiges and Onaran [20] for ways to compute these from contact surgery diagrams. 
Recall that a rationally nullhomologous knot $K$ in a 3-manifold $M$ has a rational Seifert surface (or generalized Seifert surface) which may be obtained from a properly embedded orientable connected surface $\Sigma$ in $M-\mathcal{N}(K)$ such that, when oriented, $\partial \Sigma$ is a collection of coherently oriented essential curves in the torus $\partial \mathcal{N}(K)$. The surface $\Sigma$ may then be radially extended through $\mathcal{N}(K)$ to a singular surface where $K$, the core of $\mathcal{N}(K)$, is the singular set to obtain the rational Seifert surface. It is convenient however to regard $\Sigma$ as the rational Seifert surface and we say $\chi(\Sigma)$ is the Euler characteristic of the rational Seifert surface. Observe that the multiplicity $r$ with which $\partial \Sigma$ covers $K$ is the order of $K$ in homology, $r[K]=0 \in H_{1}(M)$.

Theorem 3.2.3 For a nonloose rationally nullhomologous Legendrian knot $L$ of homological order $r$ with rational Seifert surface $\Sigma$,

$$
-\left|\operatorname{tb}_{\mathbb{Q}}(L)\right|+\left|\operatorname{rot}_{\mathbb{Q}}(L)\right| \leq-\frac{1}{r} \chi(\Sigma) .
$$

Proof We follow Etnyre's proof for integrally nullhomologous knots in [14]. The idea is to take the "Seifert cable" $L^{\prime}$ of $L$ as a set of ruling curves on a convex neighborhood of $L$. Then we have a nullhomologous link $L^{\prime}$ in the tight exterior of $L$ and may relate $\operatorname{tb}\left(L^{\prime}\right)$ to $r \operatorname{tb}_{\mathbb{Q}}(L)$ and $\operatorname{rot}\left(L^{\prime}\right)$ to $r \operatorname{rot}_{\mathbb{Q}}(L)$. An application of the Bennequin type inequality to $L^{\prime}$ will yield the desired result.

Let $\Sigma$ be a rational Seifert surface for $L$, and let $N$ be a standard convex neighborhood of $L$ such that either $L^{\prime}=\partial N \cap \Sigma$ is a collection of (Legendrian) ruling curves or a collection of Legendrian divides. In the exterior of $L$, which is tight, $L^{\prime}$ is a nullhomologous Legendrian link with Seifert surface $\Sigma^{\prime}=\Sigma-N$.

Let $\lambda$ be the homology class of a dividing curve in $\partial N$ oriented parallel to $L$ and let $\mu$ be the homology class of a meridian in $\partial N$ linking $L$ positively. Then $\left[L^{\prime}\right]=$ $r \lambda+r \operatorname{tb}_{\mathbb{Q}}(L) \mu \in H_{1}(\partial N)$. Assuming that $L^{\prime}$ is a collection of ruling curves and there are just two dividing curves, the twisting of $L^{\prime}$ with respect to $\partial N$ is then $-\frac{1}{2}\left|2 \lambda \cdot\left[L^{\prime}\right]\right|=-\left|r \operatorname{tb}_{\mathbb{Q}}(L)\right|$. Because the framings on $L^{\prime}$ induced by $\partial N$ and $\partial \Sigma^{\prime}$ are equivalent, $\operatorname{tb}\left(L^{\prime}\right)=-r\left|\mathrm{tb}_{\mathbb{Q}}(L)\right|$. If $L^{\prime}$ is a collection of Legendrian divides, then it follows that $\operatorname{tb}\left(L^{\prime}\right)=0=\operatorname{tb}_{\mathbb{Q}}(L)$.

For the rotation number, trivialize the contact planes in a neighborhood of $L$ that is a slightly larger neighborhood than $N$ by extending the unit tangent vector of $L$ to a nowhere-zero section of $\xi$. Then any Legendrian curve in $\partial N$ wrapping $n$ times positively around $L$ has zero winding with respect to this trivialization and thus its rotation number is $n$ times that of $L$. Hence $\operatorname{rot}\left(L^{\prime}\right)=r \operatorname{rot}_{\mathbb{Q}}(L)$. 
Theorem 3.2.4 For a rationally nullhomologous nonloose transverse knot $T$ of homological order $r$ with rational Seifert surface $\Sigma$,

$$
\mathrm{sl}_{\mathbb{Q}}(T) \leq-\frac{1}{r} \chi(\Sigma) .
$$

Proof Assume that $s l_{\mathbb{Q}}(T)>-\frac{1}{r} \chi(\Sigma)$. For a Legendrian approximation $L$ of $T$, we have that $\left[1\right.$, Lemma 1.2] gives $\operatorname{tb}_{\mathbb{Q}}(L)-\operatorname{rot}_{\mathbb{Q}}(L)>-\frac{1}{r} \chi(\Sigma)$ since the (positive) transverse push-off of $L$ is $T$. If $\operatorname{tb}_{\mathbb{Q}}(L) \leq 0$ then the bound of Theorem 3.2.3 fails implying that $L$ is loose. Hence $T$ is loose by Proposition 5.1.1. If $\operatorname{tb}_{\mathbb{Q}}(L)>0$ then we may perform negative stabilizations of $L$ to create $L^{\prime}$ with $\operatorname{tb}_{\mathbb{Q}}\left(L^{\prime}\right) \leq 0$. Since $L^{\prime}$ is also a Legendrian approximation of $T$, we again conclude that $T$ must be loose.

\subsection{Nonloose unknots}

A knot that bounds an embedded disk is called an unknot or a trivial knot.

Theorem 3.3.1 [11] A Legendrian unknot $L$ in an overtwisted contact manifold is loose if $\operatorname{tb}(L) \leq 0$.

Eliashberg and Fraser give a coarse (ie up to contactomorphism) classification of nonloose Legendrian unknots in overtwisted contact structures on $S^{3}$ [11]. Etnyre and Vogel independently had worked out a similar proof presented in [14]. Geiges and Onaran offer an alternative proof in [20] which extends to a classification of nonloose Legendrian rational unknots in lens spaces. They explicitly present the classification for $L(p, 1)$ and $L(5,2)$, but the technique works for any lens space.

Theorem 3.3.2 [11] On $S^{3}$, only the overtwisted contact structure $\xi_{-1}$ with Hopf invariant -1 contains a nonloose unknot. For each integer $n>0$ there is a nonloose unknot with classical invariants $(\mathrm{tb}, \mathrm{rot})=(n, n-1)$ and one with $(\mathrm{tb}, \mathrm{rot})=$ $(n,-(n-1))$. Up to contactomorphism these are the only ones.

Compare the following with [14, Corollary 2.4].

Corollary 3.3.3 Suppose an overtwisted contact manifold $(M, \xi)$ contains a nonloose Legendrian unknot $L$. Then there is a nonloose Legendrian unknot $L^{\prime}$ in $\left(S^{3}, \xi_{-1}\right)$ and a tight contact structure $\xi^{\prime}$ on $M$ such that $(M, \xi)=\left(M, \xi^{\prime}\right) \#\left(S^{3}, \xi_{-1}\right)$, where $L$ is the image of $L^{\prime}$. In particular, a nonloose unknot has classical invariants ( $\left.\mathrm{tb}, \mathrm{rot}\right)=$ $(n, \pm(n-1))$ for some positive integer $n$. 
Proof We may assume $M \not S^{3}$ since the result follows directly from Theorem 3.3.2. Thus, since $L$ is an unknot, $M-L$ is reducible and $M-L \cong M \#\left(S^{3}-L^{\prime}\right)$ for an unknot $L^{\prime}$ in $S^{3}$. Since the restriction of $(M, \xi)$ to $M-L$ is tight, by the prime decomposition of tight contact manifolds (see Colin [5]), there exists a tight contact structure $\xi^{0}$ on $M$ and a tight contact structure $\xi^{\prime}$ on $S^{3}-L^{\prime}$ so that $\left(M-L,\left.\xi\right|_{M-L}\right)=\left(M, \xi^{0}\right) \#\left(S^{3}-L^{\prime}, \xi^{\prime}\right)$. Then $\left(B^{3}-L^{\prime},\left.\xi\right|_{B^{3}-L^{\prime}}\right)$, the complement of a neighborhood of a point in $\left(S^{3}-L^{\prime}, \xi^{\prime}\right)$, is contactomorphic to a contact submanifold of $\left(M-L,\left.\xi\right|_{M-L}\right)$. As such, $\left.\xi\right|_{M-L}$ extends across $L$ to $\xi$ so that $L$ is Legendrian and thus it pulls back to an extension of $\left.\xi\right|_{B^{3}-L^{\prime}}$ on $B^{3}-L^{\prime}$ across $L^{\prime}$ to a contact structure on $B^{3}$, and hence to an extension of $\xi^{\prime}$ on $S^{3}-L^{\prime}$ to a contact structure $\xi^{\prime \prime}$ on $S^{3}$, in which $L^{\prime}$ is Legendrian. Since $(M, \xi)=\left(M, \xi^{0}\right) \#\left(S^{3}, \xi^{\prime \prime}\right)$ is overtwisted, $\xi^{\prime \prime}$ cannot be tight. Thus $L^{\prime}$ in $\left(S^{3}, \xi^{\prime \prime}\right)$ is a nonloose Legendrian unknot. Theorem 3.3.2 implies $\xi^{\prime \prime}=\xi_{-1}$ and describes the possible classical invariants for $L^{\prime}$. Hence these are the possible classical invariants for $L$ as well.

Surgery descriptions of the nonloose unknots in $\left(S^{3}, \xi_{-1}\right)$ are given by Plamenevskaya in [34, Figure 3]. Corollary 3.3.3 shows that a nonloose unknot in some other overtwisted manifold may be locally presented by one of these surgery descriptions.

\section{Depth, tension, and order for Legendrian knots}

We define two geometric invariants and one algebraic invariant of Legendrian knots in a closed overtwisted contact manifold that give measures of nonlooseness. We first address the geometric ones for unoriented knots in Section 4.1 and then consider their refinements for oriented knots in Section 4.2. Thereafter, in Section 4.3 we use the LOSS invariant of [27] to define an algebraic invariant for nullhomologous Legendrian knots.

\subsection{Depth and tension}

Definition 4.1.1 The depth, $d(L)$, of a Legendrian knot $L$ in an overtwisted contact 3 -manifold is the minimum geometric intersection number $|L \cap D|$ taken over all overtwisted disks $D$ transversally intersecting $L$. The knot $L$ is loose if and only if $d(L)=0$.

Remark 4.1.2 By Proposition 3.1.1, the depth of a nonloose knot is realized with a standard overtwisted disk. 
Definition 4.1.3 The tension, $t(L)$, of a Legendrian knot $L$ in an overtwisted contact 3 -manifold is the minimum total number of stabilizations required to make $L$ loose. The knot $L$ is loose if and only if $t(L)=0$.

The following theorem shows that tension is well-defined.

Theorem 4.1.4 There is a finite sequence of stabilizations that loosens a Legendrian knot in a closed, overtwisted manifold.

Proof Let $L$ be a Legendrian knot in the overtwisted manifold $(M, \xi)$ and let $D$ be a standard overtwisted disk transverse to $L$ so that $|L \cap D|$ is a finite set. If $L$ is disjoint from $D$, then $L$ is already loose. So assume $|L \cap D|=n>0$. Since $D$ is a standard overtwisted disk, there exists a contactomorphism $f$ of a neighborhood of $D$ with a neighborhood of $D_{\text {0т }}$. Let $\lambda$ be a radial arc in $D_{\text {от }}$ from a point of $f(L \cap D)$ to $\partial D_{\text {от }}$ that is disjoint from the origin and the other points of $f(L \cap D)$. Then there is a contactomorphism $g$ that identifies a neighborhood $\left(N=\mathcal{N}(\lambda),\left.\xi\right|_{N}\right)$ with $\left(\mathbb{R}^{3}, \xi_{\text {std }}\right)$ such that $g\left(D_{\text {От }} \cap N\right)$ is the half plane $\{y \geq 0, z=0\}, g(\lambda)$ is the $\operatorname{arc}\{x=0, y \in[0,1], z=0\}$ and $g(f(L) \cap N)$ is the line $\{x=z, y=1\}$. Then, depending on the orientation of the intersection, the positive or negative stabilization as shown in the front projection of Figure 2 reduces $|L \cap D|$ by one. (View the horizontal line as the boundary of the overtwisted disk with the disk going into the page.) In this manner, repeated stabilizations make the stabilized $L$ disjoint from $D$ and thus loosen it.

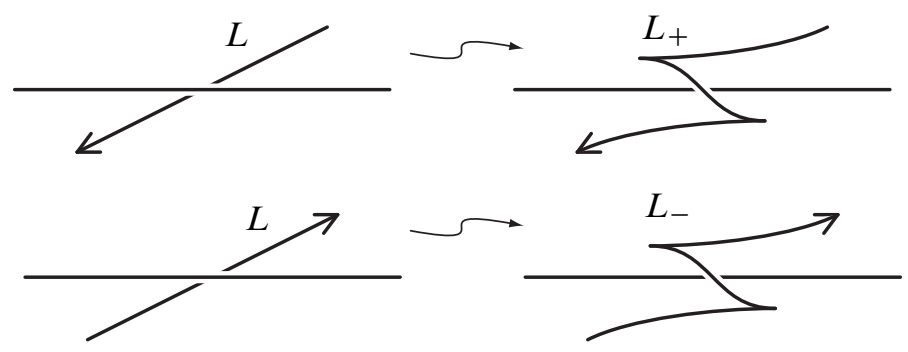

Figure 2

Remark 4.1.5 Figure 2 shows, via the front projection, how stabilizations of oriented Legendrian knots in $\left(\mathbb{R}^{3}, \xi_{\text {std }}\right)$ may remove intersections with the half plane $\{z=0, y \geq 0\}$. Orienting this half plane so that $\partial / \partial z$ is the positive normal and the boundary orientation is $\partial / \partial x$, one sees that positive stabilizations remove negative intersections while negative stabilizations remove positive intersections.

The proof of Theorem 4.1.4 offers a relationship between depth and tension. 
Lemma 4.1.6 For a Legendrian knot $L$ in a closed overtwisted contact manifold, $t(L) \leq d(L)$.

Proof Let $D$ be a standard overtwisted disk for which $|L \cap D|=d(L)$ and then apply the proof of Theorem 4.1.4. Note that a Legendrian isotopy making $L$ transverse to $D$ may be done without increasing $|L \cap D|$.

The following two theorems allow us to prove Theorem 1.0.2, that the inequality of Lemma 4.1.6 is sometimes strict.

Theorem 4.1.7 Suppose $(+1)$-surgery on a Legendrian knot $L$ with tight complement yields an overtwisted manifold with surgery dual knot $L^{*}$. Then $d\left(L^{*}\right)=1$ if and only if $L$ is a stabilization.

Proof Lemma 3.1 of [29] shows that $(+1)$-surgery on a destabilizable Legendrian knot in $\left(S^{3}, \xi_{\text {std }}\right)$ yields an overtwisted manifold. (See also the proof by Ding, Geiges and Stipsicz of [8, Theorem 1.2] as well as Ozbagci [31].) To prove this they construct an overtwisted disk that is a meridional disk of the surgery solid torus, one that the surgery dual intersects once. This proof in fact works for $(+1)$-surgery on any destabilizable knot in any contact manifold. Instead of describing framings in terms of the Thurston-Bennequin invariant, we will use the twist number.

Assume $L$ is a stabilization of a Legendrian push-off of a Legendrian knot $L^{\prime}$ in the contact manifold $(M, \xi)$. Then there is an annulus $A$ with $\partial A=L \cup L^{\prime}$ such that $\operatorname{tw}_{\xi}\left(L^{\prime}, A\right)=0$ and $\operatorname{tw}_{\xi}(L, A)=+1$. (Lisca and Stipsicz show in [29] that in $\left(S^{3}, \xi_{\text {std }}\right)$ the annulus $A$ meets $L^{\prime}$ with framing $\operatorname{tb}\left(L^{\prime}\right)$ and $L$ with framing $\operatorname{tb}(L)+1$. $)$ Then, in the contact manifold $\left(M_{+}, \xi_{+}\right)$obtained by $(+1)$-surgery on $L$, this annulus extends across a meridional disk of a solid torus neighborhood of the surgery solid torus to complete to a disk $D$. Observe that $\partial D$ is the image of $L^{\prime}$. As such, since $\mathrm{tw}_{\xi}\left(L^{\prime}, A\right)=0=\mathrm{tw}_{\xi_{+}}(\partial D, D)=\operatorname{tb}(\partial D), D$ is an overtwisted disk and the resulting manifold is overtwisted. By construction, the surgery dual knot $L^{*}$ intersects $D$ once. Thus $d\left(L^{*}\right)=1$ if the complement of $L^{*}$ is tight. The complement of $L^{*}$ is tight if and only if the complement of $L$ is tight.

Now assume $L^{*}$ is a nonloose knot in an overtwisted manifold $\left(M_{+}, \xi_{+}\right)$with $d\left(L^{*}\right)=1$. Then, by Proposition 3.1.1, there is a standard overtwisted disk $D$ which $L^{*}$ intersects once. Then, in the contact manifold $(M, \xi)$ obtained by $(-1)-$ surgery on $L^{*}, D-L^{*}$ extends to an annulus $A$ whose boundary is the image of $\partial D$ and the surgery dual to $L^{*}$, Legendrian knots $L^{\prime}$ and $L$ respectively. Furthermore $\mathrm{tw}_{\xi}\left(L^{\prime}, A\right)=\mathrm{tw}_{\xi_{+}}(\partial D, D)=\operatorname{tb}(\partial D)=0$ and, due to performing $(-1)$-surgery 
on $L^{*}, \operatorname{tw}_{\xi}(L, A)=+1$. Hence the contact planes of $\xi$ have nonpositive twisting along each component of $\partial A=L \cup L^{\prime}$ relative to the framing by $A$. Therefore we may realize $A$ as a convex surface with dividing set $\Gamma$ consisting of a possibly empty set of simple closed curves and a single arc with its endpoints in $L$. Since this arc of $\Gamma$ is boundary parallel in $A$, it signifies that $L$ is a stabilization (see eg Etnyre [13, Lemma 2.20]).

Theorem 4.1.8 Suppose $(+1)$-surgery on a Legendrian knot $L$ in $\left(S^{3}, \xi_{\text {std }}\right)$ yields an overtwisted manifold with surgery dual knot $L^{*}$. If $\operatorname{tb}(L)<-1, \operatorname{rot}(L)<0$, and $\operatorname{tb}(L)+\operatorname{rot}(L)+2<\chi(L)$, then $t\left(L^{*}\right)=1$.

Proof Assume $\left(M_{+}, \xi_{+}\right)$is the overtwisted manifold produced by $(+1)-$ surgery on $L$. Let $L^{\prime}$ be a Legendrian push-off of $L$. Then the image of $L^{\prime}$ in $\left(M_{+}, \xi_{+}\right)$is a Legendrian push-off of the surgery dual knot $L^{*}$. Hence the link $L \cup L^{\prime}$ with $(+1)-$ surgery on $L$ is a surgery diagram for $L^{*}$ in $\left(M_{+}, \xi_{+}\right)$. Similarly, $L \cup L_{+}^{\prime}$ with $(+1)$-surgery on $L$ is a surgery diagram for the stabilization $L_{+}^{*}$ of $L^{*}$ in $\left(M_{+}, \xi_{+}\right)$.

Given that $\left(M_{+}, \xi_{+}\right)$is overtwisted by assumption and that $L$ is a Legendrian knot in $S^{3}, L^{*}$ is a nonloose knot. Hence $t\left(L^{*}\right)>0$. To show that $t\left(L^{*}\right)=1$ we will demonstrate that the rational classical bound for nonloose knots, Theorem 3.2.3, does not hold for a positive stabilization of $L^{*}$ if $-\chi(L)<-(\operatorname{rot}(L)+\operatorname{tb}(L)+2)$. To do so, we will calculate $\operatorname{tb}_{\mathbb{Q}}\left(L_{+}^{*}\right)$ and $\operatorname{rot}_{\mathbb{Q}}\left(L_{+}^{*}\right)$ using the methods in [20].

Observe that $(+1)$-surgery on $L$ is a topological $\operatorname{tb}(L)+1$ - surgery on $L$; hence the homological order $r$ of $L^{*}$ is $|\operatorname{tb}(L)+1|$. Moreover, because $\operatorname{tb}(L) \neq-1$ by assumption, this means that $M_{+}$is a rational homology sphere and [20, Lemma 2] will apply. If $\Sigma$ is a rational Seifert surface for $L_{+}^{*}$ of minimal genus, then topologically it is the image of a minimal genus Seifert surface for $L$. Hence $\chi(L)=\chi(\Sigma)$. Also note that $\ell k\left(L, L_{+}^{\prime}\right)=\ell k\left(L, L^{\prime}\right)=\operatorname{tb}(L)$ since $L^{\prime}$ is a Legendrian push-off of $L$ and $L_{+}^{\prime}$ is topologically isotopic to $L^{\prime}$ in the complement of $L$. Following [20, Lemma 2], let $M=(\operatorname{tb}(L)+1)$ be the linking matrix of $L$ and $M_{0}=\left(\begin{array}{cc}0 & \operatorname{tb}(L) \\ \operatorname{tb}(L) & \operatorname{tb}(L)+1\end{array}\right)$ be the extended matrix. Then we may compute that

$$
\begin{aligned}
\operatorname{tb}_{\mathbb{Q}}\left(L_{+}^{*}\right)= & \operatorname{tb}\left(L_{+}^{\prime}\right)+\frac{\operatorname{det} M_{0}}{\operatorname{det} M}=\operatorname{tb}(L)-1+\frac{-\operatorname{tb}^{2}(L)}{\operatorname{tb}(L)+1}=\frac{-1}{\operatorname{tb}(L)+1}, \\
\operatorname{rot}_{\mathbb{Q}}\left(L_{+}^{*}\right) & =\operatorname{rot}\left(L_{+}^{\prime}\right)-\left\langle\operatorname{rot}(L), M^{-1}(\operatorname{tb}(L))\right\rangle \\
& =\operatorname{rot}(L)+1-\left\langle\operatorname{rot}(L), \frac{1}{\operatorname{tb}(L)+1} \cdot \operatorname{tb}(L)\right\rangle \\
& =\operatorname{rot}(L)+1-\frac{\operatorname{rot}(L) \operatorname{tb}(L)}{\operatorname{tb}(L)+1}=\frac{\operatorname{rot}(L)+(\operatorname{tb}(L)+1)}{\operatorname{tb}(L)+1},
\end{aligned}
$$


where $\operatorname{tb}\left(L_{+}^{\prime}\right)=\operatorname{tb}(L)-1$ and $\operatorname{rot}\left(L_{+}^{\prime}\right)=\operatorname{rot}(L)+1$ since $L_{+}^{\prime}$ is a positive stabilization of $L$.

Assume that $L_{+}^{*}$, a positive stabilization of $L^{*}$, is nonloose. Then by Theorem 3.2.3 it holds that

$$
\begin{aligned}
-\left|\operatorname{tb}_{\mathbb{Q}}\left(L_{+}^{*}\right)\right|+\left|\operatorname{rot}_{\mathbb{Q}}\left(L_{+}^{*}\right)\right| & \leq-\frac{1}{r} \chi(\Sigma), \\
-\left|\frac{-1}{\operatorname{tb}(L)+1}\right|+\left|\frac{\operatorname{rot}(L)+\operatorname{tb}(L)+1}{\operatorname{tb}(L)+1}\right| & \leq-\frac{1}{|\operatorname{tb}(L)+1|} \chi(L), \\
\frac{1}{\operatorname{tb}(L)+1}+\frac{\operatorname{rot}(L)+\operatorname{tb}(L)+1}{\operatorname{tb}(L)+1} & \leq \frac{\chi(L)}{\operatorname{tb}(L)+1}, \\
\operatorname{rot}(L)+\operatorname{tb}(L)+2 & \geq \chi(L),
\end{aligned}
$$

since $\operatorname{rot}(L)<0$ and $\operatorname{tb}(L)+1<0$. But this contradicts our assumption that $-(\operatorname{rot}(L)+\operatorname{tb}(L)+2)>-\chi(L)$. Hence $L_{+}^{*}$ is loose and thus $t\left(L^{*}\right)=1$.

In the introduction, we sketched a proof of Theorem 1.0.2 which asserts that there exists nonloose Legendrian knots $L$ with $1=t(L)<d(L)$. We now provide a more detailed proof. Observe that the knots constructed are only rationally nullhomologous.

Proof of Theorem 1.0.2 Assume that $L$ is a nondestabilizable Legendrian knot in $\left(S^{3}, \xi_{\text {std }}\right)$ satisfying $\operatorname{tb}(L) \leq-2, \operatorname{rot}(L)<0,-\chi(L)<-(\operatorname{rot}(L)+\operatorname{tb}(L)+2)$ and $(+1)$-surgery on $L$ is overtwisted. Let $L^{*}$ be the Legendrian knot dual to the $(+1)-$ surgery on $L$. Since $L$ and $L^{*}$ have contactomorphic complements, $L^{*}$ is a nonloose knot. Because $L$ is nondestabilizable, Theorem 4.1.7 implies that $d\left(L^{*}\right) \geq 2$. Due to the assumptions on $\operatorname{tb}(L), \operatorname{rot}(L)$ and $\chi(L)$, Theorem 4.1.8 implies $t\left(L^{*}\right)=1$. Now we simply need to confirm that such Legendrian knots exist in $\left(S^{3}, \xi_{\text {std }}\right)$.

Let $p$ and $q$ be coprime integers satisfying $-p>q>0$. By [15, Theorem 4.1] the maximal Thurston-Bennequin invariant of Legendrian representatives of the negative torus knot $T_{p, q}$ is $p q$. By [15, Theorem 4.4], among these Legendrian representatives with maximal Thurston-Bennequin invariant is one with rotation number $q+p$. Take $L$ to be this torus knot; hence $\operatorname{tb}(L)=p q<0, \operatorname{rot}(L)=q+p<0$ and $\chi(L)=$ $|q|-|p||q-1|=q-p+p q$. Since $0>2(p+1)$, it follows that $-\chi(L)<-(\operatorname{rot}(L)+$ $\operatorname{tb}(L)+2)$. Finally, $(+1)$-surgery on $L$ is overtwisted by [29, Corollary 1.2].

For nonloose Legendrian unknots, the depth and tension are always equal to 1.

Lemma 4.1.9 If $L$ is a nonloose Legendrian unknot in an overtwisted manifold, then $t(L)=d(L)=1$. 
Proof Theorem 4.1.7 applies to the surgery descriptions of the nonloose Legendrian unknots in $\left(S^{3}, \xi_{-1}\right)$ given in [34] (see also [20]) to show that these knots all have depth 1. (More explicitly, each nonloose Legendrian unknot is presented in a surgery description of $\left(S^{3}, \xi_{-1}\right)$ as a Legendrian push-off of a stabilized Legendrian unknot on which $(+1)$-surgery is done; as such, the nonloose Legendrian unknot is Legendrian isotopic to the surgery dual of that component.) It then follows from Corollary 3.3.3 that the surgery diagrams of [34] may be used with Theorem 4.1.7 to show that a nonloose Legendrian unknot in any overtwisted manifold also has depth 1. Lemma 4.1.6 then implies any nonloose Legendrian unknot also has tension 1.

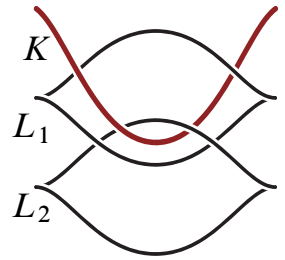

(a)

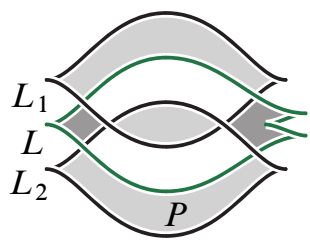

(b)

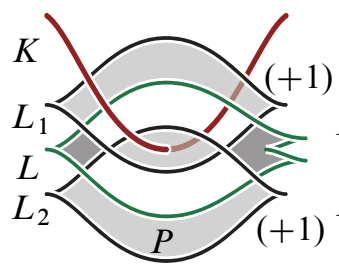

(c)

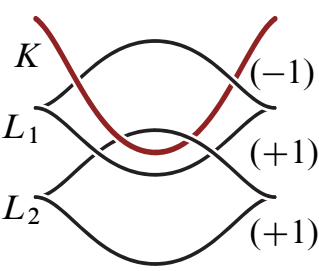

(d)

Figure 3

Proposition 4.1.10 Let $K$ be a Legendrian knot in $(M, \xi)$ with standard Legendrian meridian $L_{1}$ and its Legendrian push-off $L_{2}$, locally pictured as in the front diagram of Figure 3(a). The result of $(+1)$-surgeries on both $L_{1}$ and $L_{2}$ is the overtwisted manifold $\left(M^{\prime}, \xi^{\prime}\right)=(M, \xi) \#\left(S^{3}, \xi_{-1}\right)$. Letting $K^{\prime}$ be the image of $K$ in $\left(M^{\prime}, \xi^{\prime}\right)$, then $d\left(K^{\prime}\right) \leq 1$. Furthermore, if either $(M, \xi)$ or $(+1)$-surgery on $K$ is tight then $d\left(K^{\prime}\right)=1$.

Proof Let us first focus locally on the link $L_{1} \cup L_{2}$ of Legendrian unknots of $t \mathrm{~b}=-1$ with their $(+1)$-surgeries. Viewed in $\left(S^{3}, \xi_{\text {std }}\right)$, this pair of surgeries yields $\left(S^{3}, \xi_{-1}\right)$ giving the first claim. We may construct an overtwisted disk in $\left(M^{\prime}, \xi^{\prime}\right)$ as follows: Figure 3(b) adds a third Legendrian unknot $L$ of $\mathrm{tb}=-2$ which cobounds a thricepunctured sphere $P$ with $L_{1} \cup L_{2}$. Observe that $P$ meets $L_{1}$ and $L_{2}$ with topological framing 0 and $L$ with the contact framing. Hence, after the pair of $(+1)$-surgeries, $P$ caps off to a disk $D$ where the boundary of $D$ is (the image of) $L$. Since $P$ meets $L$ with the contact framing, $D$ is an overtwisted disk.

Now including $K$ in our consideration, we see that we may choose $L$ and $P$ so that $K$ intersects $P$ once as in Figure 3(c). Thus $K^{\prime}$, the image of $K$ in $\left(M^{\prime}, \xi^{\prime}\right)$, intersects the overtwisted disk $D$ once. Hence $d\left(K^{\prime}\right) \leq 1$.

If $(-1)$-surgery on $K^{\prime}$ produces a tight manifold, then $K^{\prime}$ will be nonloose so that $d\left(K^{\prime}\right)=1$. Locally, a surgery diagram for this manifold is given in Figure 3(d). By 
Ding and Geiges [7, Proposition 2], since $L_{1}$ is a standard Legendrian meridians of $K$, it is Legendrian isotopic to a Legendrian push-off of $K$ after the $(-1)$-surgery on $K$. Since $L_{2}$ is a Legendrian push-off of $L_{1}$, it has a Legendrian isotopy following that of $L_{1}$ as a push-off. Thus, after $(-1)$-surgery on $K, L_{1} \cup L_{2}$ is Legendrian isotopic to a Legendrian push-off of $K$ with a further push-off. Thus $(+1)$-surgery on $L_{1}$, say, cancels the $(-1)$-surgery on $K$ returning the manifold $(M, \xi)$ in which $L_{2}$ is now both the only remaining surgery curve and Legendrian isotopic to the original knot $K$. Hence the result of $(-1)$-surgery on $K^{\prime}$ in $\left(M^{\prime}, \xi^{\prime}\right)$ may also be obtained by $(+1)$-surgery on $K$ in $(M, \xi)$.

Remark 4.1.11 Observe that the surgery duals to (-1)-surgery on $K^{\prime}$ and $(+1)-$ surgery on $K$ are Legendrian isotopic knots. Therefore from the conclusion of Proposition 4.1.10, Theorem 4.1.7 implies $d\left(K^{\prime}\right)=1$ if and only if the surgery dual to $(+1)$-surgery on $K$ is a stabilization.

Lemma 4.1.12 (Proof of [28, Theorem 1.1]) If $K$ is a Legendrian knot in $\left(S^{3}, \xi_{\text {std }}\right)$ with $\operatorname{tb}(K)=2 g_{s}(K)-1>1$, then $(+1)$-surgery on $K$ is tight.

Here $g_{s}(K)$ denotes the smooth 4-ball genus of a knot $K$ in $S^{3}$.

Example 4.1.13 Let $p, q$ be positive coprime integers. Let $K$ be a Legendrian $(p, q)-$ torus knot in $\left(S^{3}, \xi_{\text {std }}\right)$ with $\operatorname{tb}(K)=p q-p-q=2 g_{s}(K)-1$. (Such Legendrian knots do exist; see [15].) Thus by Proposition 4.1.10 and Lemma 4.1.12, (+1)surgeries on a standard Legendrian meridian and its push-off send $K$ to a Legendrian $(p, q)$-torus knot in $\left(S^{3}, \xi_{-1}\right)$ with depth 1 .

Example 4.1.14 An upper bound on tension may be obtained from a violation of Theorem 3.2.1 after some number of stabilizations. Here we show that there are nonloose Legendrian knots $K$ with $t(K)=1$ for which Theorem 3.2.1 only offers a large upper bound.

Continuing with the previous example, Figure 4 gives an explicit surgery diagram for nonloose Legendrian $(2, q)$-torus knots $L_{2, q}$ in $\left(S^{3}, \xi_{-1}\right)$ that have depth and tension equal to 1. A straightforward computation with the formula from [27, Lemma 6.6] shows the invariants of these nonloose torus knots are $\operatorname{tb}\left(L_{2, q}\right)=q$ and $\operatorname{rot}\left(L_{2, q}\right)=0$. So after $q$ stabilizations this knot will violate the inequality in Theorem 3.2.1 thereby only implying that $t\left(L_{2, q}\right) \leq q$.

Theorem 4.1.7 may be generalized to give characterizations of Legendrian knots of larger depth. Topologically, if a knot $K^{*}$ transversally intersects the interior of a disk $D$ 


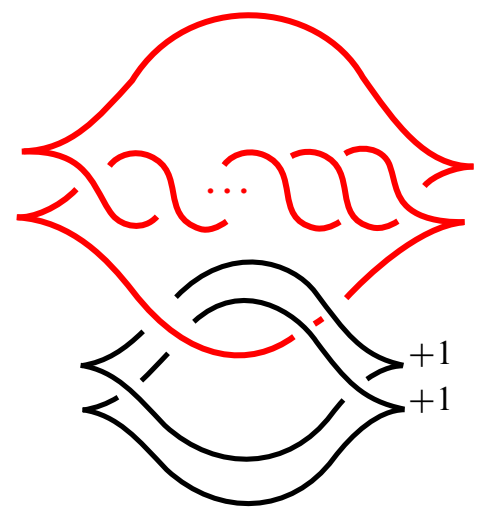

Figure 4: Nonloose $(2, q)$-torus knots in $\left(S^{3}, \xi-1\right)$

a total of $n$ times, then each of these intersections may be tubed to a meridian $m^{*}$ of $K^{*}$ creating a "folded surface" $\Sigma$ where the "boundary" of $\Sigma$ is $\partial D$ together with $n$ copies of $m^{*}$. (The terminology "folded" comes from Turaev [37]. When the algebraic intersection number of $K^{*}$ with $D$ is $\pm n$, then this folded surface is a rational Seifert surface for the link $\partial D \cup m^{*}$.) Being disjoint from $K^{*}$, this folded surface $\Sigma$ persists through surgery on $K^{*}$. Moreover, after integral surgery on $K^{*}$, the surgery dual knot $K$ is isotopic to $m^{*}$ and hence $\Sigma$ is a folded surface for the link $\partial D \cup K$ with multiplicity 1 on $\partial D$ and multiplicity $n$ on $K$.

In Theorem 4.1.15 below, we examine the above construction in the contact setting for $n=2$ where the folded surface $\Sigma$ is an honest surface, a once-punctured torus or Klein bottle.

Theorem 4.1.15 Suppose $(+1)$-surgery on a Legendrian knot $L$ in $(M, \xi)$ with tight complement yields an overtwisted manifold with surgery dual knot $L^{*}$. Then $d\left(L^{*}\right)=2$ if and only if:

(1) $L$ is not a stabilization.

(2) There is a once-punctured torus or once-punctured Klein bottle $\Sigma$ with $\partial \Sigma$ Legendrian and $\operatorname{tw}_{\xi}(\partial \Sigma, \Sigma)=0$ that contains $L$ as an essential, nonseparating, orientation-preserving curve in $\Sigma$ with $\operatorname{tw}_{\xi}(L, \Sigma)=+1$.

Note that while every isotopy class of essential, simple closed curves in a once-punctured torus is nonseparating and orientation preserving, there is a unique such class in a once-punctured Klein bottle. 
Proof This proceeds in the same manner as the proof Theorem 4.1.7.

Assume $L$ is a curve in a surface $\Sigma$ as in part (2) of the hypotheses so that $\Sigma-L$ is a thrice-punctured sphere. Then $(+1)$-contact surgery on $L$ topologically compresses $\Sigma$ to a disk $D$ that $L^{*}$ intersects twice, that is, $(+1)$-contact surgery fills the exterior of $L$ to cap off two boundary components of $\Sigma-\mathcal{N}(L)$. Since $\partial D=\partial \Sigma, D$ is an overtwisted disk. Hence $d\left(L^{*}\right) \leq 2$. Because $L$ is not a stabilization by (1) but has tight complement, $d\left(L^{*}\right) \geq 2$ by Theorem 4.1.7. Thus $d\left(L^{*}\right)=2$.

Now $L^{*}$ is a Legendrian knot in the overtwisted manifold $\left(M_{+}, \xi_{+}\right)$and assume $d\left(L^{*}\right)=2$. Let $D$ be a standard overtwisted disk that $L^{*}$ intersects twice transversally. Take a tb $=-1$ Legendrian meridian $m^{*}$ of $L^{*}$ so that $L^{\prime}$ is isotopic to the Legendrian knot dual to $(-1)$-surgery on $L^{*}$. Then tube $D$ along an arc of $L^{*}$ through $m^{*}$ to form $\Sigma$, a once-punctured torus or once-punctured Klein bottle (depending whether or not $L^{*}$ has trivial algebraic intersection number with $D$ ). Observe that $\operatorname{tw}_{\xi_{+}}\left(m^{*}, \Sigma\right)=+1$ by construction. If $L$ is the surgery dual knot and $(M, \xi)$ is the resulting contact manifold, then since $\xi$ and $\xi_{+}$agree on the complements of neighborhoods of $L$ and $L^{*}$, we may Legendrian isotope $L$ into the position of $m^{*}$ so that $\operatorname{tw}_{\xi}(L, \Sigma)=+1$. By construction $\operatorname{tw}_{\xi}(\partial \Sigma, \Sigma)=0$ and $L$ is not a stabilization by Theorem 4.1.7.

Remark 4.1.16 As of this writing, we have yet to use Theorem 4.1.15 to construct any explicit examples of Legendrian knots $L^{*}$ of depth 2 arising as surgery duals to knots in $\left(S^{3}, \xi_{\text {std }}\right)$. Presumably such knots exist. The knots we were able to construct satisfying (2) of Theorem 4.1.15 ended up being stabilizations.

One approach is to begin with a genus- 1 Legendrian knot $K$ in a tight $(M, \xi)$ with $\operatorname{tb}(K)=0$. Then $K$ has a once-punctured torus Seifert surface $\Sigma$ which may be made convex with dividing set $\Gamma$ disjoint from $K=\partial \Sigma$. Assuming $\Gamma$ has just two (parallel) components, let $L$ be an essential curve in $\Sigma$ that intersects each component of $\Gamma$ once. We may assume $L$ is Legendrian by the Legendrian realization principle (see Kanda [26] and Honda [23]) and thus conclude that $\operatorname{tw}_{\xi}(L, \Sigma)=+1$. The only thing left to check is whether or not $L$ is a stabilized knot.

Another approach is to begin with a nonstabilized Legendrian knot $L$ in a tight $(M, \xi)$ (or just with tight complement) for which $(+1)$-contact surgery is overtwisted and attempt to construct the surface $\Sigma$ of Theorem 4.1.15. Take a pair of Legendrian push-offs and insert an extra twist to form an annulus $A$ that contains $L$ with $\partial A$ Legendrian and each of these three curves having $\operatorname{tw}_{\xi}(\bullet, A)=+1$. Then the trick is to find a Legendrian banding of the components of $\partial A$ that results in a surface $\Sigma$ with $\operatorname{tw}_{\xi}(\partial \Sigma, \Sigma)=0$. 


\subsection{Orientation refinements}

As defined, depth and tension do not depend upon the orientation of a Legendrian knot. Nevertheless, if a nonloose knot is oriented, one may care to keep track of the oriented intersections with an overtwisted disk and the signs of stabilizations that loosen it. Recall from Section 3.1 that an overtwisted disk $D$ is oriented so that $\operatorname{rot}(\partial D)>0$ and from Remark 4.1.5 that a positive stabilization removes a negative intersection of an oriented Legendrian knot with a standard overtwisted disk while a negative stabilization removes a positive intersection.

Definition 4.2.1 Let $L$ be an oriented Legendrian knot in an overtwisted contact structure.

Define $d_{\circ}(L)$, the oriented depth of $L$, to be the set of pairs $(p, n)$ such that $L$ transversally intersects some overtwisted disk $D$ positively $p$ times and negatively $n$ times with $p+n=d(L)$.

Define $t_{\circ}(L)$, the oriented tension of $L$, to be the set of pairs $(a, b)$ such that the stabilization $L_{+a,-b}$ is loose and $a+b=t(L)$.

Definition 4.2.2 Let $L$ be an oriented Legendrian knot in an overtwisted contact structure. Define $t_{+}(L)$, the positive tension of $L$, to be the minimum number of positive stabilizations required to loosen $L$ if possible, and $\infty$ otherwise. Similarly define $t_{-}(L)$, the negative tension of $L$. Observe that $t_{ \pm}(-L)=t_{\mp}(L)$ where $-L$ is $L$ with its orientation reversed.

Due to their relationship to the LOSS invariant and relevance for transverse knots, we choose to focus upon positive and negative tensions instead of the oriented depth and tension.

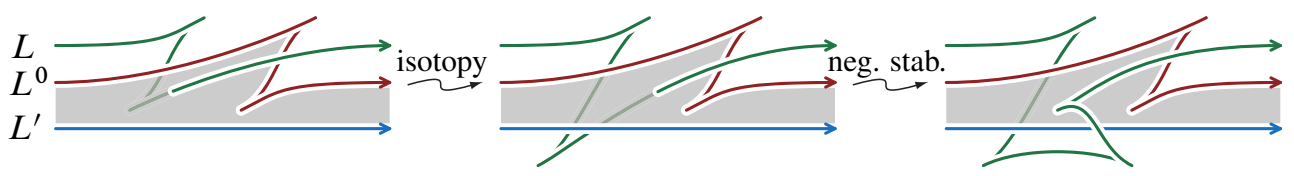

Figure 5

We may partially extend Theorem 4.1 .7 for these signed tensions.

Proposition 4.2.3 Assume the oriented Legendrian knot $L$ in the contact manifold $(M, \xi)$ is a positive stabilization of the Legendrian knot $L^{\prime}$ and has tight complement. Let $L^{*}$ be the oriented knot surgery dual to $(+1)$-surgery on $L$.

Then $t_{-}\left(L^{*}\right)=1$. Furthermore, if the Heegaard Floer contact invariant for $(+1)-$ surgery on $L^{\prime}$ in $(M, \xi)$ is nonzero, then $t_{+}\left(L^{*}\right)>1$. 
Ozsváth and Szabó's Heegaard Floer contact invariant is defined in [32]; its behavior under connected sums and $(-1)$-contact surgery follows from this.

Proof Let $L$ be a positive stabilization of a Legendrian push-off of a Legendrian knot $L^{\prime}$ and let $A$ be the annulus between them. Let $L^{0}$ be a further Legendrian push-off of $L$. This situation is illustrated in Figure 5 on the left. It further indicates how a negative stabilization of $L^{0}$ can remove the intersection with $A$.

As in Theorem 4.1.7 and [29, Lemma 3.1], after $(+1)$-surgery on $L, L^{0}$ is Legendrian isotopic to the surgery dual knot $L^{*}$ and $A$ caps off to an overtwisted disk $D$ with $\partial D=L^{\prime}$. Thus a negative stabilization on $L^{*}$ can remove the intersection with $D$, and so $t_{-}\left(L^{*}\right)=1$.

We will now show that $(-1)$-surgery on a positive stabilization $L_{+}^{*}$ of $L^{*}$ has nonzero contact invariant if $(M, \xi)$ does too in order to conclude that $L_{+}^{*}$ is nonloose and hence $t_{+}\left(L^{*}\right)>1$. To do so, we will first construct an open book supporting the contact manifold obtained by $(-1)$-surgery on $L_{+}^{*}$ and relate it to open books supporting $(M, \xi)$ and $(+1)$-surgery on $L^{\prime}$.

Let $(\Sigma, \phi)$ be an (abstract) open book supporting $(M, \xi)$ in which the page $\Sigma$ contains the Legendrian knot $L^{\prime}$ as a nonseparating curve so that the page framing agrees with the contact framing. Giroux shows how to do this in [22]. We may assume $\phi$ acts trivially in a collar neighborhood of $\partial \Sigma$. Let $a$ be an embedded arc in $\Sigma$ with one endpoint on $\partial \Sigma$, the other endpoint on $L^{\prime}$ and interior disjoint from $L^{\prime}$. A neighborhood of $a$ is shown in Figure 6(a). Let $A$ be a regular annular neighborhood of $L^{\prime} \cup a$. We will be modifying $(\Sigma, \phi)$ within this neighborhood.

A single stabilization of this open book near $a \cap \partial \Sigma$ allows the Legendrian knot $L$ (a positive stabilization of $L^{\prime}$ ) to embed in this stabilized page with page framing equal to its contact framing. This is shown in Figure 6(b) along with a parallel copy $L^{0}$ of $L$. A further stabilization of the open book then lets $L_{+}^{0}$, a positive stabilization of $L^{0}$, embed in the page with page framing equal to its contact framing as shown in Figure 6(c). Figure 7(a) shows this again in the entirety of $A$ with the two stabilizations. Let $\left(\Sigma^{\prime \prime}, \partial_{2} \circ \partial_{1} \circ \phi^{\prime \prime}\right)$ denote this resulting twice-stabilized open book for $(M, \xi)$. Since the stabilizations occur in a neighborhood of a point in $\partial \Sigma$, we may view $\Sigma^{\prime \prime}$ as a subsurface of $\Sigma$ whose complement is two open disks in a neighborhood of $a$ (as opposed to viewing $\Sigma^{\prime \prime}$ as the result of attaching two 1-handles to $\Sigma$ ) and $\phi^{\prime \prime}$ is the restriction of $\phi$ to $\Sigma^{\prime \prime}$. The maps $\partial_{1}$ and $\partial_{2}$ indicate positive Dehn twists along a curve parallel to each of the two new boundary components.

Let $\phi^{*}=\tau_{L}^{-1} \circ \partial_{2} \circ \partial_{1}$ and $\phi_{+}^{*}=\tau_{L_{+}^{0}} \circ \phi^{*}$. It now follows that $\left(\Sigma^{\prime \prime}, \phi^{*} \circ \phi^{\prime \prime}\right)$ is an open book for $\left(M_{+}, \xi_{+}\right)$and $\left(\Sigma^{\prime \prime}, \phi_{+}^{*} \circ \phi^{\prime \prime}\right)$ is an open book for $(-1)$-surgery on $L_{+}^{*}$. 
Let $A^{\prime \prime}=A \cap \Sigma^{\prime \prime}$, a four-punctured sphere. (Notice that $L^{\prime}$ and $L_{+}^{0}$ are isotopic in $A^{\prime \prime}$ to two of the components of $\partial A^{\prime \prime}$. The other two boundary components come from the stabilizations.) Observe that $\phi_{+}^{*}$ is the identity outside of $A^{\prime \prime}$ and within $A^{\prime \prime}$ may be represented as in Figure 7(b). By the lantern relation, we may express $\phi_{+}^{*}$ as $\tau_{\gamma} \circ \tau_{\beta} \circ \tau_{L^{\prime}}^{-1}$ as shown in Figure 7(c) where $\gamma$ and $\beta$ are the two curves in the middle. (Only one choice of which is which is correct for the order of composition of Dehn twists, but our proof does not require this detail.) Thus $\left(\Sigma^{\prime \prime}, \tau_{\gamma} \circ \tau_{\beta} \circ \tau_{L^{\prime}}^{-1} \circ \phi^{\prime \prime}\right)$ is an open book for $(-1)$-surgery on $L_{+}^{*}$.

Let $\left(M^{\prime}, \xi^{\prime}\right)$ denote the result of $(+1)$-surgery on $L^{\prime}$ in $(M, \xi)$, and observe that $\left(\Sigma, \tau_{L^{\prime}}^{-1} \circ \phi\right)$ is an open book for this manifold. Hence $\left(\Sigma^{\prime \prime}, \tau_{L^{\prime}}^{-1} \circ \phi^{\prime \prime}\right)$ is an open book for $\left(M^{\prime}, \xi^{\prime}\right) \#\left(S^{1} \times S^{2}, \xi_{\text {std }}\right) \#\left(S^{1} \times S^{2}, \xi_{\text {std }}\right)$. Since $\left(M^{\prime}, \xi^{\prime}\right)$ has nonzero Heegaard Floer contact invariant by assumption, so does $\left(M^{\prime}, \xi^{\prime}\right) \#\left(S^{1} \times S^{2}, \xi_{\text {std }}\right)$ \# $\left(S^{1} \times S^{2}, \xi_{\text {std }}\right)$. Then adding positive Dehn twists along $\beta$ and $\gamma$ to the monodromy of the open book $\left(\Sigma^{\prime \prime}, \tau_{L^{\prime}}^{-1} \circ \phi^{\prime \prime}\right)$ supporting this manifold results in an open book supporting a contact structure that also has nonzero Heegaard Floer contact invariant by Baldwin [2]. Therefore, since (-1)-surgery on $L_{+}^{*}$ is supported by the open book $\left(\Sigma^{\prime \prime}, \tau_{\gamma} \circ \tau_{\beta} \circ \tau_{L^{\prime}}^{-1} \circ \phi^{\prime \prime}\right)$, the contact manifold is tight. Consequently $L_{+}^{*}$ is a nonloose knot in $\left(M_{+}, \xi_{+}\right)$and $t_{+}\left(L^{*}\right)>1$.

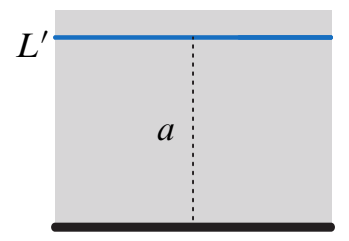

(a)

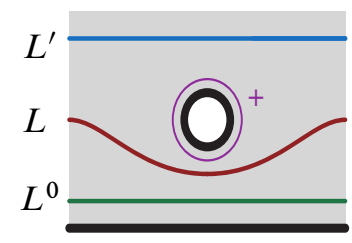

(b)

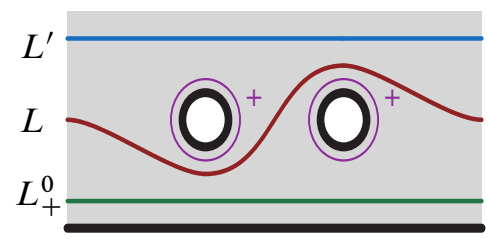

(c)

Figure 6

Example 4.2.4 Using Proposition 4.2.3 one may construct many examples of nonloose Legendrian knots with $t_{-}=1$ and $t_{+}>1$.

Let $(M, \xi)$ be a contact manifold resulting from $(-1)$-surgeries on each component of an oriented Legendrian link $L_{0} \cup L_{1} \cup \cdots \cup L_{k}$ in $\left(S^{3}, \xi_{\text {std }}\right)$, and let $L^{\prime}$ the surgery dual knot to $L_{0}$. Then $(+1)$-surgery on $L^{\prime}$ is the result of $(-1)$-surgeries on the sublink $L_{1} \cup \cdots \cup L_{k}$, a Stein fillable contact manifold which therefore has a nonzero Heegaard Floer contact invariant [32]. Now let $L$ be a positive stabilization of $L^{\prime}$ in $(M, \xi)$. Then let $\left(M_{+}, \xi_{+}\right)$be the overtwisted manifold resulting from $(+1)$-surgery on $L$ and let $L^{*}$ be the surgery dual. According to Proposition 4.2.3, $t_{-}\left(L^{*}\right)=1$ and $t_{+}\left(L^{*}\right)>1$. 


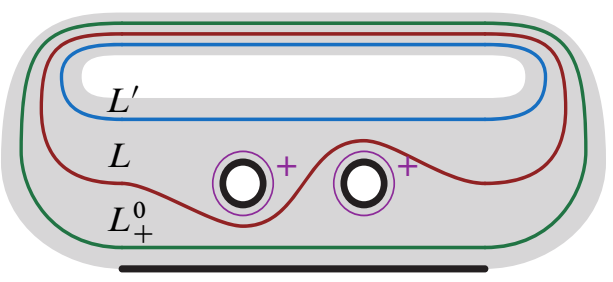

(a)

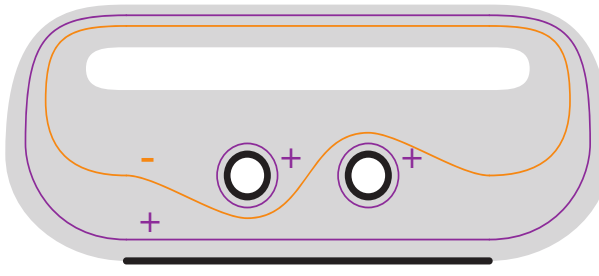

(b)

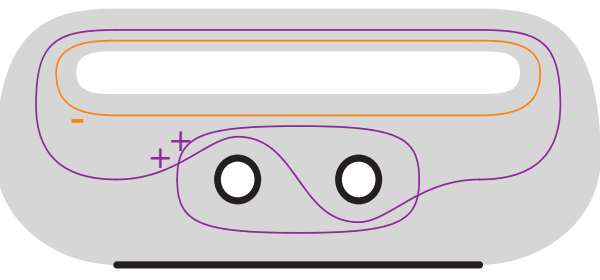

(c)

Figure 7

Example 4.2.5 By Corollary 3.3.3, a nonloose unknot has ( $\mathrm{tb}, \mathrm{rot})=(n, \pm(n-1))$ for some positive integer $n$. Therefore if $L$ is a nonloose unknot oriented so that $\operatorname{rot}(L)=1-n$ with $n>0, t_{-}(L)=1$ and $t_{+}(L) \leq n$. If $n \geq 2$, then applying Proposition 4.2.3 as described in Example 4.2.4 to the (local) surgery descriptions of [34, Figure 3] shows that $t_{+}(L) \geq 2$. Thus $t_{\circ}(L)=\{(1,0),(0,1)\}$ if $n=1$ and $t_{\circ}(L)=\{(0,1)\}$ if $n \geq 2$.

Example 4.2.6 In [20], Geiges and Onaran extend Plamenevskaya's surgery descriptions of nonloose Legendrian unknots in $\left(S^{3}, \xi_{-1}\right)$ to nonloose Legendrian rational unknots (ie knots with solid torus exterior) in overtwisted lens spaces. They explicitly give surgery diagrams for such rational unknots in the lens spaces $L(p, 1)$ for $p \in \mathbb{N}$ and in $L(5,2)$. Following Example 4.2.4, we may apply Proposition 4.2.3 to show the nonloose knots described in [20, Figures 6(b)(c), 7(b)(c) and 8(a)(b)(c)] may be oriented to have $t_{-}=1$ and $t_{+} \geq 2$.

\subsection{Order and the LOSS invariant}

Lisca, Ozsváth, Stipsicz and Szabó define an invariant $\mathfrak{L}$ which is a $U$-torsion element in $H_{F K^{-}}(-M, L)$ of an oriented, nullhomologous Legendrian knot $L$ in an overtwisted contact manifold $(M, \xi)$. As is common, we often refer to $\mathfrak{L}$ as the LOSS invariant. Lemmas 1.4 and 1.6 of [27] show that $\mathfrak{L}$ enjoys the following properties:

- If $L$ is loose, then $\mathfrak{L}(L)=0$.

- If $L_{-}$is a negative stabilization of $L$, then $\mathfrak{L}\left(L_{-}\right)=\mathfrak{L}(L)$.

- If $L_{+}$is a positive stabilization of $L$, then $\mathfrak{L}\left(L_{+}\right)=U \cdot \mathfrak{L}(L)$. 
The following definition is suggested by [27, Corollary 1.3].

Definition 4.3.1 Let $L$ be an oriented, nullhomologous Legendrian knot in an overtwisted manifold. Define $o(L)$ to be the order of the $U$-torsion of $\mathfrak{L}(L)$, that is

$$
o(L)=\min \left\{k \in \mathbb{N}: U^{k} \cdot \mathfrak{L}(L)=0\right\} .
$$

Definition 4.3.2 Let $L$ be an unoriented, nullhomologous Legendrian knot in an overtwisted contact structure. Then the order of $L$ is $\bar{o}(L)=o(L)+o(-L)$, the sum of the orders of its two orientations (where an arbitrary orientation on $L$ is chosen for the calculation).

Lemma 4.3.3 Let $L$ be an oriented nullhomologous Legendrian knot in an overtwisted contact structure. Given a set of $a$ positive stabilizations and $b$ negative stabilizations such that $L_{+a,-b}$ is loose, then

$$
o(L) \leq a \quad \text { and } \quad o(-L) \leq b .
$$

Proof The result of $b$ negative stabilizations of $L$ is $L_{-b}$ and $\mathfrak{L}\left(L_{-b}\right)=\mathfrak{L}(L)$. Then $L_{+a,-b}$ is the result of $a$ positive stabilizations of $L_{-b}$. Hence $\mathfrak{L}\left(L_{+a,-b}\right)=$ $U^{a} \cdot \mathfrak{L}\left(L_{-b}\right)=U^{a} \cdot \mathfrak{L}(L)$. Since $L_{+a,-b}$ is loose, $\mathfrak{L}\left(L_{+a,-b}\right)=0$. Therefore $o(L) \leq a$. Reversing the orientation on $L$, the other result follows similarly.

Lemma 4.3.4 If $L$ is a nullhomologous Legendrian knot in an overtwisted contact structure then

$$
\bar{o}(L) \leq t(L)
$$

Proof Apply Lemma 4.3.3 to any partition $a+b$ of $t(L)$ so $L_{+a,-b}$ is loose.

Lemma 4.3.5 Let $L$ be a nonloose, nullhomologous, oriented Legendrian knot. If $\mathfrak{L}(L) \neq 0$, then all its negative stabilizations are nonloose. That is, $o(L)>0$ implies $t_{-}(L)=\infty$. Moreover, $L$ must intersect every overtwisted disk negatively.

Proof Since negative stabilizations do not change $\mathfrak{L}$, the first statement holds. Since positive intersections of an oriented Legendrian knot with an overtwisted disk may be removed by negative stabilizations (Remark 4.1.5) and such a knot $L$ with $\mathfrak{L}(L) \neq 0$ cannot be loosened by only negative stabilizations, there must be a negative intersection of $L$ with any overtwisted disk. 
Example 4.3.6 Let $\xi_{n}$ be the overtwisted contact structure on $S^{3}$ with Hopf invariant $h\left(\xi_{n}\right)=1-2 n$. For each $n \in \mathbb{N}$, [27, Lemma 6.1] gives a family of nonloose Legendrian representatives $L(n)$ of the $(2,2 n+1)$-torus knots in $\left(S^{3}, \xi_{n}\right)$. Together [27, Proposition 6.2 and Remark 6.3] show that $\mathfrak{L}(L(n)) \neq 0$; hence $o(L(n))>0$ and, as in Lemma 4.3.5, $t_{-}(L(n))=\infty$.

Since stabilizations decrease $t b$, it follows that for each $n \in \mathbb{N}$ the values of $t b$ of the nonloose Legendrian representatives of the $(2,2 n+1)$-torus knots in $\left(S^{3}, \xi_{n}\right)$ are not bounded below.

Proposition 4.3.7 If a nullhomologous knot type $\mathcal{K}$ has a lower bound on the ThurstonBennequin invariants of its nonloose Legendrian representatives in a given overtwisted manifold, then $\mathfrak{L}(L)=0, o(L)=0, \bar{o}(L)=0$ and $t_{ \pm}(L)<\infty$ for each (oriented) Legendrian representative $L$.

Proof Given any oriented Legendrian representative $L$ of $\mathcal{K}$, sufficiently many negative stabilizations will produce a representative $L^{\prime}$ with $\operatorname{tb}\left(L^{\prime}\right)$ less than the assumed lower bound. Hence $L^{\prime}$ must be loose. Thus $t_{-}(L)<\infty, \mathfrak{L}(L)=\mathfrak{L}\left(L^{\prime}\right)=0$ and so $o(L)=0$. A similar argument shows $t_{+}(L)<\infty, o(-L)=0$, and thus $\bar{o}(L)=0$.

Corollary 4.3.8 Any Legendrian unknot $L$ in an overtwisted contact structure has $\bar{o}(L)=0$.

Proof By Theorem 3.3.1, any nonloose Legendrian unknot has $t b>0$.

Remark 4.3.9 For any Legendrian knot $L$ in a contact manifold $(M, \xi)$, Sivek [35] defines a monopole Floer invariant $\ell_{g}(L) \in \operatorname{KHM}(-M, L)$ for each integer $g \geq 2$ which one may care to compare with the Heegaard Floer LOSS invariant $\mathfrak{L}(L) \in$ $H_{F K}^{-}(M, L)$. These invariants $\ell_{g}$ are all 0 if either the complement of $L$ is overtwisted or $L=L_{+1,-1}^{\prime}$ for some other Legendrian knot $L^{\prime}$ with any orientation. As such, while these $\ell_{g}$ may be able to detect nonlooseness, they appear to be less suited for bounding tension.

We caution the reader that [35, Proposition 5.6] contains an error in the penultimate sentence of its proof. This also impacts [35, Corollary 5.7]. Furthermore the knots constructed in the example following Corollary 5.7 and illustrated in Figure 7 of that article are in fact all loose since their surgery descriptions involve $(+1)$-surgery on a stabilized trefoil. Consequently, examples of nonloose Legendrian knots in overtwisted manifolds with nonzero $\ell_{g}$ have yet to be produced. 


\section{Depth, tension, and order for transverse knots}

\subsection{Transverse knots and their Legendrian approximations}

Recall the following key facts from Section 2.2.3. Throughout, all knots are oriented.

- Any two Legendrian approximations of a transverse knot are related by negative stabilizations.

- The transverse push-off of a Legendrian approximation is equivalent to the original transverse knot.

- Any invariant of Legendrian knots that is unaltered by negative stabilization is an invariant of transverse knots.

Indeed, a transverse knot has only one kind of stabilization. It may be viewed as the transverse push-off of a positive stabilization of a Legendrian approximation of the original transverse knot.

We define the invariants depth, tension and order for transverse knots in the obvious manner. Corollary 5.1.5(1) below shows that tension is well defined. Since the LOSS invariant $\mathfrak{L}$ of a nullhomologous Legendrian knot is unaltered by negative stabilization, the order of a nullhomologous transverse knot is defined to be the order of any Legendrian approximation.

Proposition 5.1.1 Let $L$ be a Legendrian knot in an overtwisted contact structure. Then $L$ is a Legendrian approximation of a nonloose transverse knot if and only if $t_{-}(L)=\infty$. Also $t_{-}(L)<\infty$ if and only if the transverse push-off of $L$ is loose.

Remark 5.1.2 This sharpens [14, Proposition 1.2] by clarifying when a transverse push-off of a nonloose Legendrian knot is loose.

Proof Since transverse push-offs and Legendrian approximations are inverse operations up to negative stabilizations, the two statements of the proposition are equivalent. We will prove the second. First assume $L$ is a Legendrian knot with $t_{-}(L)<\infty$. Then $t_{-}(L)$ negative stabilizations to $L$ produces a loose Legendrian knot $L^{\prime}$. Since $L$ and $L^{\prime}$ have the same transverse push-off, it must be loose.

Now assume the transverse push-off $T$ of $L$ is loose. Then there is an overtwisted disk disjoint from a neighborhood of $T$. Hence some Legendrian approximation $L^{\prime}$ of $T$ (taken within this neighborhood of $T$ ) must be loose. But since $L$ and $L^{\prime}$ must have common negative stabilizations, some negative stabilization of $L$ is loose. Thus $t_{-}(L)<\infty$. 
Example 5.1.3 Example 4.2.4 gives a procedure to create examples of Legendrian knots with $t_{-}=1$. Proposition 5.1.1 implies each of these knots has a loose transverse push-off.

Remark 5.1.4 Lemma 4.3.5 shows a nonloose, nullhomologous oriented Legendrian knot $L$ with $\mathfrak{L}(L) \neq 0$ (and hence $o(L)>0$ ) implies $t_{-}(L)=\infty$. Proposition 5.1.1 then implies the transverse push-off of $L$ is nonloose. That $\mathfrak{L}(L) \neq 0$ implies $t_{-}(L)=\infty$ and the transverse push-off of $L$ is nonloose is effectively the content of the end of the proof of [27, Corollary 7.3].

Proposition 5.1.1 has several corollaries.

Corollary 5.1.5 (1) There is a finite sequence of stabilizations that loosens a nonloose transverse knot.

(2) Positive stabilizations are required to loosen any Legendrian approximation of a nonloose transverse knot.

(3) If the transverse push-off of a (rationally) nullhomologous Legendrian knot is nonloose, then there is no lower bound on the (rational) Thurston-Bennequin invariant for nonloose Legendrian representatives of this knot type.

(4) A transverse unknot in an overtwisted contact structure is loose [14, Corollary 2.3].

Proof (1) By Theorem 4.1.4 a Legendrian approximation of a transverse knot may be loosened by a finite sequence of stabilizations. Then the transverse push-off of this loosened Legendrian approximation is loose by Proposition 5.1.1. The positive stabilizations used in loosening the Legendrian approximation correspond to the loosening stabilizations of the original transverse knot.

(2) This is immediate.

(3) Negative stabilizations must remain nonloose, but each such stabilization decreases the (rational) Thurston-Bennequin invariant. Indeed, by Proposition 4.3.7, if there were a lower bound then $t_{-}<\infty$ for any Legendrian approximation.

(4) If not, then by (3) there would be no lower bound on tb for nonloose Legendrian unknot, contrary to Theorem 3.3.1.

Lemma 5.1.6 If $T$ is a nonloose transverse knot and $L$ is a Legendrian approximation, then $t(T) \leq t(L)$. 
Proof Every stabilization of a transverse knot corresponds to a positive stabilization of its Legendrian approximations. If $L$ is loosened by $p$ positive stabilizations and $n$ negative stabilizations so that $t(L)=p+n$, then $t(T) \leq p \leq t(L)$.

Lemma 5.1.7 Assume a Legendrian approximation $L$ of a nonloose transverse knot $T$ requires at least one positive stabilization and at least one negative stabilization to loosen. Then $0<t(T)<t(L)$.

Proof As $T$ is transversally isotopic to the transverse push-off of all negative stabilizations of $L$, it is loosened by only the positive stabilizations.

\subsection{Bindings of overtwisted open books}

An open book induces a contact structure in which the binding is naturally a transverse link.

Lemma 5.2.1 If a knot $T$ is the connected binding of an open book decomposition that supports an overtwisted contact structure, then $T$ is a nonloose transverse knot in that contact structure.

Proof More is actually true. The complement of the binding (connected or not) of an open book for any contact manifold is universally tight. A proof of this fact appears in the proof of [17, Lemma 3.1] by Etnyre and Vela-Vick.

Let $H^{+}$and $H^{-}$respectively denote the positive and negative Hopf bands in $S^{3}$. The open book $\left(S^{3}, H^{+}\right)$whose page is $H^{+}$supports the tight contact structure on $S^{3}$. The open book $\left(S^{3}, H^{-}\right)$whose page is $H^{-}$supports the overtwisted contact structure $\xi_{-1}$ (with Hopf invariant -1 ) on $S^{3}$. An open book is a positive or negative Hopf stabilization if it may be obtained by plumbing such a Hopf band onto another open book.

Recall that if an open book $(M, \Sigma)$ supports the contact structure $\xi$, then $\left(M, \Sigma \sharp_{\alpha} H^{+}\right)$, the positive Hopf stabilization obtained by plumbing a positive Hopf band onto the page $\Sigma$ of $(M, \Sigma)$ along an arc $\alpha \subset \Sigma$ also supports $\xi$ [22]. In particular, if $\alpha$ is a boundary-parallel arc in $\Sigma, \Sigma \sharp_{\alpha} H^{+}$is the boundary connected sum of the page $\Sigma$ and the band $\mathrm{H}^{+}$. Indeed, this plumbing may be positioned so that the supported contact structure is identical rather than merely isotopic to the original. Consequently, the binding of the resulting open book in $\xi$ is the same transverse link $\partial \Sigma$ with the addition of a transverse unknot encircling as a meridional curve the component of $\partial \Sigma$ along which the Hopf band was summed. A local picture of this is illustrated in Figure 8. 
$\Sigma$

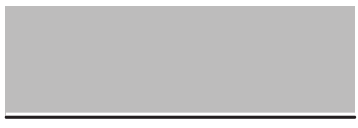

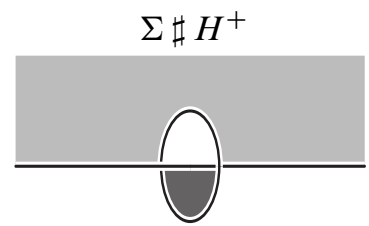

Figure 8

Lemma 5.2.2 Let $H^{-} \sharp H^{+}$denote the boundary connected sum of a negative and a positive Hopf band. The open book $\left(S^{3}, H^{-} \sharp H^{+}\right)$supports a contact structure containing an overtwisted disk $D$ such that $\partial D$ is a 0 -framed loop in $H^{-} \sharp H^{+}$that is parallel to one component of $\partial\left(H^{-} \sharp H^{+}\right)$and the interior of $D$ intersects each of the other two components just once.

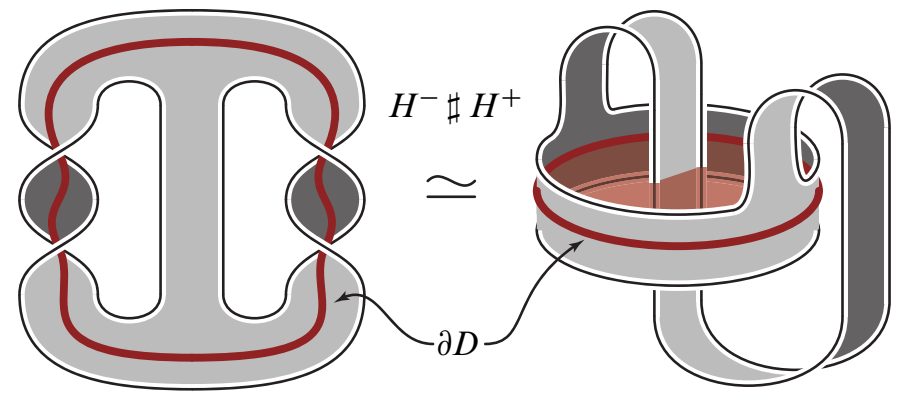

Figure 9

Proof Figure 9 shows the surface $H^{-} \sharp H^{+}$with the curve $\partial D$ on the left and an isotopic presentation of this surface together with the disk $D$ on the right. Let $\Sigma$ be the union of $\mathrm{H}^{-} \sharp H^{+}$with another page of the open book which we may then assume is convex such that the binding is the dividing set; see Torisu [36]. Since $\partial D$ is not the sole boundary component of a subsurface of $H^{-} \sharp H^{+}$it may be realized as a Legendrian curve for which $t_{w}(\partial D, \Sigma)$ equals its page-framing in the supported contact structure $[26 ; 23]$. Since the page framing is the Seifert framing for $\partial D$ and $\partial D$ is disjoint from the dividing set, $\operatorname{tb}(\partial D)=0$. Hence $D$ is an overtwisted disk with the properties claimed.

Theorem 5.2.3 Assume an open book with connected binding is a negative Hopf stabilization. Then the binding $T$, as the nonloose transverse knot in the overtwisted contact structure the open book supports, has $d(T)=t(T)=1$.

Proof Let $(M, \Sigma)$ be a negatively Hopf stabilized open book for $M$ with page $\Sigma$ and connected binding $T=\partial \Sigma$ that supports the contact structure $\xi$. By Lemma 5.2.1, $T$ 
is a nonloose transverse knot and thus $d(T)>0$. By assumption we have $(M, \Sigma)=$ $\left(M, \Sigma_{0} \sharp_{\beta} H^{-}\right)$, the plumbing of the negative Hopf band onto the open book $\left(M, \Sigma_{0}\right)$ along an arc $\beta \subset \Sigma_{0}$. Plumb the positive Hopf band onto $(M, \Sigma)$ along a boundary parallel arc $\alpha \subset \Sigma$ to form the open book $\left(M, \Sigma \sharp_{\alpha} H^{+}\right)$; that is, take the boundary connected sum of $\Sigma$ with $\mathrm{H}^{+}$. Since this is a positive stabilization of the original open book $(M, \Sigma)$, it supports the same contact structure $\xi$. As discussed above, the binding of $\left(M, \Sigma \sharp_{\alpha} H^{+}\right)$is the link $T \cup \mu$ consisting of the transverse knot $T$ and a transverse meridian (as pictured in Figure 8).

We now find an overtwisted disk whose interior is intersected twice by $T \cup \mu$, once by each. This will exhibit a depth 1 overtwisted disk for $T$. The boundary connect sum of $H^{+}$with $\Sigma$ may be done at a point on $\partial H^{-}$disjoint form $\Sigma_{0}$. Then we may view $\left(M, \Sigma \sharp_{\alpha} H^{+}\right)$as the plumbing of $\left(S^{3}, H^{-} \sharp H^{+}\right)$onto $\left(M, \Sigma_{0}\right)$ along a rectangle in $H^{-} \sharp H^{+}$disjoint from $H^{+}$. With this plumbing, the overtwisted disk $D$ of Lemma 5.2.2 carries over to an overtwisted disk in $\left(M, \Sigma \sharp_{\alpha} H^{+}\right)$where its boundary is a 0 -framed curve in the page and its interior intersects each $T$ and $\mu$ just once. Hence $d(T)=1$.

Remark 5.2.4 Depth (and tension) can be defined for links. We can extend the proof of Theorem 5.2.3 easily to negatively Hopf stabilized open books with bindings of multiple components, showing that there is an overtwisted disk intersected just once by the binding. As such, Theorem 5.2.3 may be viewed as offering an obstruction to an open book being a negative Hopf stabilization.

Example 5.2.5 Let $T_{1} \cup T_{2}=\partial H^{-}$be the transverse link arising as the binding of the open book $\left(S^{3}, H^{-}\right)$in the supported overtwisted contact structure $\xi_{-1}$. By Lemma 5.2.1 the link is nonloose. However since $T_{1}$ and $T_{2}$ are both unknots, individually they are loose transverse knots according to Corollary 5.1.5(4). This implies that $T_{1}$ intersects every overtwisted disk that is disjoint from $T_{2}$ and $T_{2}$ intersects every overtwisted disk that is disjoint from $T_{1}$. Theorem 5.2.3, extended for disconnected binding, implies $d\left(T_{1} \cup T_{2}\right)=1$.

Remark 5.2.6 Ito and Kawamuro use their theory of open book foliations to view overtwistedness of a contact structure through a "transverse overtwisted disk" with respect to an open book supporting the contact structure [24; 25]. Such disks give an upper bound on the depth of the binding of the open book; see [24, Proposition 4.2].

\section{Problems and questions}

Problem 6.1 Develop constructions of knots of large depth or tension. 
Topological operations on knots and links often have Legendrian analogues. It is natural to ask how our invariants of depth and tension behave under these operations. Two fundamental operations are Legendrian Whitehead doubles (see Fuchs [18], Ng and Traynor [30] and Etnyre [13]) and Legendrian cables (see Etnyre and Honda [16] and Ding and Geiges [6]). While some sources only explicitly define these operations for Legendrian knots in $\left(\mathbb{R}^{3}, \xi_{\text {std }}\right)$, their definitions of other contact manifolds are straightforward.
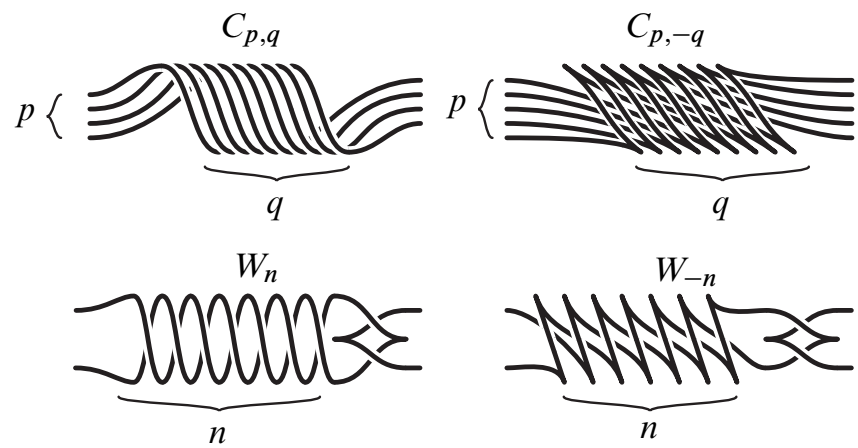

Figure 10: Legendrian cables and twisted Whitehead doubles for the $x$-axis in the front projection, modulo translation

For coprime integers $p, q$ with $p>0$, let $C_{p, q}(L)$ denote the Legendrian $(p, q)$ cable of the Legendrian knot $L$. For an integer $n$, let $W_{n}(L)$ denote the $n$-twisted Legendrian Whitehead double of the Legendrian knot $L$. These satellites, taken in a standard contact solid torus neighborhood of $L$, are illustrated in Figure 10.

Question 6.2 For a nonloose Legendrian knot $L$, how are the depth, tension and order of $L$ and $C_{p, q}(L)$ related? In particular, given coprime positive integers $p$ and $q$, does $d\left(C_{p, q}(L)\right)=p \cdot d(L)$ ? When is $C_{p, q}(L)$ loose?

Question 6.3 For a nonloose Legendrian knot $L$, how are the depth, tension and order of $L$ and $W_{n}(L)$ related? In particular, if $n \geq 0$ does $d\left(W_{n}(L)\right)=2 \cdot d(L)$ ? When is $W_{n}(L)$ loose?

Problem 6.4 Construct explicit examples of Legendrian knots $L$ with $d(L)=2$.

Of course Theorems 4.1.7 and 4.1.15 as well as Remark 4.1.16 should be kept in mind. The previous two questions suggest investigating the cables $C_{2, q}\left(L^{*}\right)$ and Whitehead doubles $W_{n}\left(L^{*}\right)$ of the $(+1)$-surgery duals to a stabilized knot. 
Question 6.5 Do the $(+1)$-surgery duals to the Legendrian knots in $\left(S^{3}, \xi_{\text {std }}\right)$ having a local configuration as in [29, Figure 1] all have depth at most 2? In particular, do the surgery duals to $(+1)$-surgery on negative torus knots with maximal ThurstonBennequin invariant all have depth 2?

Lisca and Stipcisz show that $(+1)$-contact surgery on these knots produce an overtwisted contact structure by constructing a once-punctured torus with Legendrian boundary in the surgered manifold that violates the Bennequin-type inequality for tight contact structures. Being duals to knots in a tight manifold, they are necessarily nonloose. At the very least one should be able to determine an upper bound on depth for all such knots (such as in the discussion preceding Theorem 4.1.15).

Problem 6.6 Study the discrepancy between depth and tension.

(1) How big can $d-t$ be among Legendrian knots?

(2) Does every knot type have an upper bound on $d-t$ for its Legendrian representatives in each overtwisted contact structure?

(3) Is there a nullhomologous Legendrian knot with $d-t>0$ ?

While the proof of Theorem 1.0.2 only exhibits rationally nullhomologous Legendrian knots with $d-t>0$, there should be nullhomologous examples. Indeed, we expect that every overtwisted manifold contains nonloose Legendrian knots for which this difference can be arbitrarily large, though not within a single knot type.

Question 6.7 Can both the positive and negative tension of a nonloose Legendrian knot be large?

(1) Is there a nonloose Legendrian knot $L$ for which $t_{+}(L)$ and $t_{-}(L)$ are both bigger than $t(L)$ ? That is, is there a nonloose Legendrian knot which requires both a positive and a negative stabilization to realize its tension?

(2) Are there nonloose Legendrian knots with both $t_{+}=\infty$ and $t_{-}=\infty$ ? A Legendrian Whitehead double of a knot with $t_{-}=\infty$ seems like a likely candidate.

Problem 6.8 Determine the overtwisted contact structures and knot types for which there is a lower bound on the Thurston-Bennequin invariant of the nonloose Legendrian representatives.

If such a knot type is rationally nullhomologous, then Corollary 5.1.5(3) together with Proposition 5.1.1 implies every transverse representative is loose and $t_{-}<\infty$ for each Legendrian representative. If it is actually nullhomologous, then by Proposition 4.3.7 $\mathfrak{L}=0$ and $o=0$ as well. 
Question 6.9 If an open book with connected binding $K$ supports an overtwisted contact structure, must $t(K)=1$ ?

We expect the answer to be no. For comparison, Theorem 5.2.3 says that $t(K)=$ $d(K)=1$ whenever the open book admits a negative stabilization.

Problem 6.10 Extend the LOSS invariant to rationally nullhomologous knots, and study the order of rationally nullhomologous nonloose knots.

Question 6.11 Are there nonloose transverse knots for which every Legendrian approximation has $\mathfrak{L}=0$ ?

Problem 6.12 Find a nonloose Legendrian knot with nonzero $\ell_{g}$; see Remark 4.3.9. The nonloose unknots in $\left(S^{3}, \xi_{-1}\right)$ are reasonable candidates.

Recall that we have been working under the assumption that our overtwisted manifolds are closed. The definitions of depth and tension clearly extend to overtwisted manifolds with boundary where some overtwisted disk is contained in the interior of the manifold.

However, if every overtwisted disk in an overtwisted contact manifold with boundary were properly embedded, then stabilizations could not loosen a nonloose knot. Indeed Vela-Vick pointed out that Theorem 4.1.4 fails if $(V, \xi)$ is the contact solid torus $V=\{(r, \theta, z): r \leq \pi\} /(z \mapsto z+1)$ with $\xi=\operatorname{ker}(\cos r d z+r \sin r d \theta)$. Any Legendrian knot in $V$ not contained in a ball is necessarily nonloose.

Problem 6.13 Characterize overtwisted manifolds with boundary in which every overtwisted disk is properly embedded.

\section{References}

[1] K Baker, J Etnyre, Rational linking and contact geometry, from: "Perspectives in analysis, geometry and topology", (I Itenberg, B Jöricke, M Passare, editors), Progr. Math. 296, Springer, New York (2012) 19-37 MR2884030

[2] J A Baldwin, Comultiplicativity of the Ozsváth-Szabó contact invariant, Math. Res. Lett. 15 (2008) 273-287 MR2385640

[3] D Bennequin, Entrelacements et équations de Pfaff, from: "Third Schnepfenried geometry conference, Vol. 1”, Astérisque 107, Soc. Math. France, Paris (1983) 87-161 MR753131

[4] V Chernov, Framed knots in 3-manifolds and affine self-linking numbers, J. Knot Theory Ramifications 14 (2005) 791-818 MR2172898 
[5] V Colin, Chirurgies d'indice un et isotopies de sphères dans les variétés de contact tendues, C. R. Acad. Sci. Paris Sér. I Math. 324 (1997) 659-663 MR1447038

[6] F Ding, H Geiges, Legendrian knots and links classified by classical invariants, Commun. Contemp. Math. 9 (2007) 135-162 MR2313510

[7] F Ding, H Geiges, Handle moves in contact surgery diagrams, J. Topol. 2 (2009) 105-122 MR2499439

[8] F Ding, H Geiges, A I Stipsicz, Surgery diagrams for contact 3-manifolds, Turkish J. Math. 28 (2004) 41-74 MR2056760

[9] K Dymara, Legendrian knots in overtwisted contact structures arXiv: math/0410122

[10] Y Eliashberg, Legendrian and transversal knots in tight contact 3-manifolds, from: "Topological methods in modern mathematics", (L R Goldberg, A V Phillips, editors), Publish or Perish, Houston, TX (1993) 171-193 MR1215964

[11] Y Eliashberg, M Fraser, Topologically trivial Legendrian knots, J. Symplectic Geom. 7 (2009) 77-127 MR2496415

[12] J Epstein, D Fuchs, M Meyer, Chekanov-Eliashberg invariants and transverse approximations of Legendrian knots, Pacific J. Math. 201 (2001) 89-106 MR1867893

[13] J B Etnyre, Legendrian and transversal knots, from: "Handbook of knot theory", (W Menasco, M Thistlethwaite, editors), Elsevier, Amsterdam (2005) 105-185 MR2179261

[14] J B Etnyre, On knots in overtwisted contact structures, Quantum Topol. 4 (2013) 229-264 MR3073563

[15] J B Etnyre, K Honda, Knots and contact geometry, I: Torus knots and the figure eight knot, J. Symplectic Geom. 1 (2001) 63-120 MR1959579

[16] J B Etnyre, K Honda, Cabling and transverse simplicity, Ann. of Math. 162 (2005) 1305-1333 MR2179731

[17] J B Etnyre, D S Vela-Vick, Torsion and open book decompositions, Int. Math. Res. Not. 2010 (2010) 4385-4398 MR2737776

[18] D Fuchs, Chekanov-Eliashberg invariant of Legendrian knots: Existence of augmentations, J. Geom. Phys. 47 (2003) 43-65 MR1985483

[19] H Geiges, An introduction to contact topology, Cambridge Studies in Adv. Math. 109, Cambridge Univ. Press (2008) MR2397738

[20] H Geiges, S Onaran, Legendrian rational unknots in lens spaces, J. Symp. Geom. 13 (2015) 17-50

[21] E Giroux, Convexité en topologie de contact, Comment. Math. Helv. 66 (1991) 637-677 MR1129802

[22] E Giroux, Géométrie de contact: de la dimension trois vers les dimensions supérieures, from: "Proceedings of the International Congress of Mathematicians, Vol. II", (T Li, editor), Higher Ed. Press, Beijing (2002) 405-414 MR1957051 
[23] K Honda, On the classification of tight contact structures, I, Geom. Topol. 4 (2000) 309-368 MR1786111

[24] T Ito, K Kawamuro, Open book foliation, Geom. Topol. 18 (2014) 1581-1634 MR3228459

[25] T Ito, K Kawamuro, Visualizing overtwisted discs in open books, Publ. Res. Inst. Math. Sci. 50 (2014) 169-180 MR3167583

[26] Y Kanda, The classification of tight contact structures on the 3-torus, Comm. Anal. Geom. 5 (1997) 413-438 MR1487723

[27] P Lisca, P Ozsváth, A I Stipsicz, Z Szabó, Heegaard Floer invariants of Legendrian knots in contact three-manifolds, J. Eur. Math. Soc. (JEMS) 11 (2009) 1307-1363 MR2557137

[28] P Lisca, A I Stipsicz, Ozsváth-Szabó invariants and tight contact three-manifolds, I, Geom. Topol. 8 (2004) 925-945 MR2087073

[29] P Lisca, A I Stipsicz, Notes on the contact Ozsváth-Szabó invariants, Pacific J. Math. 228 (2006) 277-295 MR2274521

[30] L Ng, L Traynor, Legendrian solid-torus links, J. Symplectic Geom. 2 (2004) 411-443 MR2131643

[31] B Ozbagci, A note on contact surgery diagrams, Internat. J. Math. 16 (2005) 87-99 MR2115679

[32] P Ozsváth, Z Szabó, Heegaard Floer homology and contact structures, Duke Math. J. 129 (2005) 39-61 MR2153455

[33] F Öztürk, Generalised Thurston-Bennequin invariants for real algebraic surface singularities, Manuscripta Math. 117 (2005) 273-298 MR2154251

[34] O Plamenevskaya, On Legendrian surgeries between lens spaces, J. Symplectic Geom. 10 (2012) 165-181 MR2926993

[35] S Sivek, Monopole Floer homology and Legendrian knots, Geom. Topol. 16 (2012) 751-779 MR2928982

[36] I Torisu, Convex contact structures and fibered links in 3-manifolds, Internat. Math. Res. Notices (2000) 441-454 MR1756943

[37] V Turaev, A function on the homology of 3-manifolds, Algebr. Geom. Topol. 7 (2007) 135-156 MR2308939

Department of Mathematics, University of Miami

1365 Memorial Drive, Coral Gables, FL 33124-4250, USA

Department of Mathematics, Hacettepe University

06800 Beytepe-Ankara, Turkey

k.baker@math.miami.edu, sonaran@hacettepe.edu.tr

Received: 21 May $2014 \quad$ Revised: 18 August 2014 